Supporting information

\title{
Mobility-Classified Mass Spectrometry Reveals a \\ Complete Picture of the Electrospray Outcome
}

Christian Lübbert*, Wolfgang Peukert

*Institute of Particle Technology (LFG), Friedrich-Alexander University Erlangen Nuremberg, Cauerstraße 4, 91058 Erlangen (Germany)

35 pages, 41 figures, 1 table 


\section{S1: DMA-MS and SMPS results for the dilution series of lysozyme}

For the protein dilution series $72.5 \mathrm{mg}$ of lysozyme were dissolved in $50 \mathrm{ml}$ of the water/formic acid stock solution which results, considering a protein mass of $14300 \mathrm{Da}$, in a concentration of $101 \mu \mathrm{mol} / \mathrm{l}$. Based on this solution a dilution series employing dilution ratios of either 1:1 or 2:3 was made. The realized dilution ratios and theoretical concentrations are given in table 1.

Table 1: Dilution ratios and theoretically resulting concentrations used in the dilution experiment and the theoretically expected most abundant clusters in the parent droplet evaporation residues (figure 6 in the manuscipt) as derived from droplet size and concentration.

\begin{tabular}{|l|l|l|l|l|l|l|l|l|l|l|l|l|}
\hline Dilution ratio & & $1: 1$ & $2: 3$ & $2: 3$ & $2: 3$ & $2: 3$ & $2: 3$ & $2: 3$ & $1: 1$ & $1: 1$ & $1: 1$ & $1: 1$ \\
\hline $\mathrm{c} /(\mu \mathrm{mol} / \mathrm{I})$ & 101 & 51 & 34 & 23 & 15 & 10 & 6.7 & 4.5 & 2.2 & 1.1 & 0.55 & 0.28 \\
\hline $\mathrm{N}_{\text {max,theo. }}$ & 122 & 63 & 42 & 28 & 18 & 12 & 8 & 5 & 2 & 1 & 0 & 0 \\
\hline
\end{tabular}

Figures 1 a-l shows the positive and negative ion mode mobility-classified mass spectra for the complete dilution series. The corresponding size measurements by SMPS are given in figures s3 a-1. From the cumulative volume distributions one can be read out the fraction of single molecules with respect to the total amount of lysozyme. 

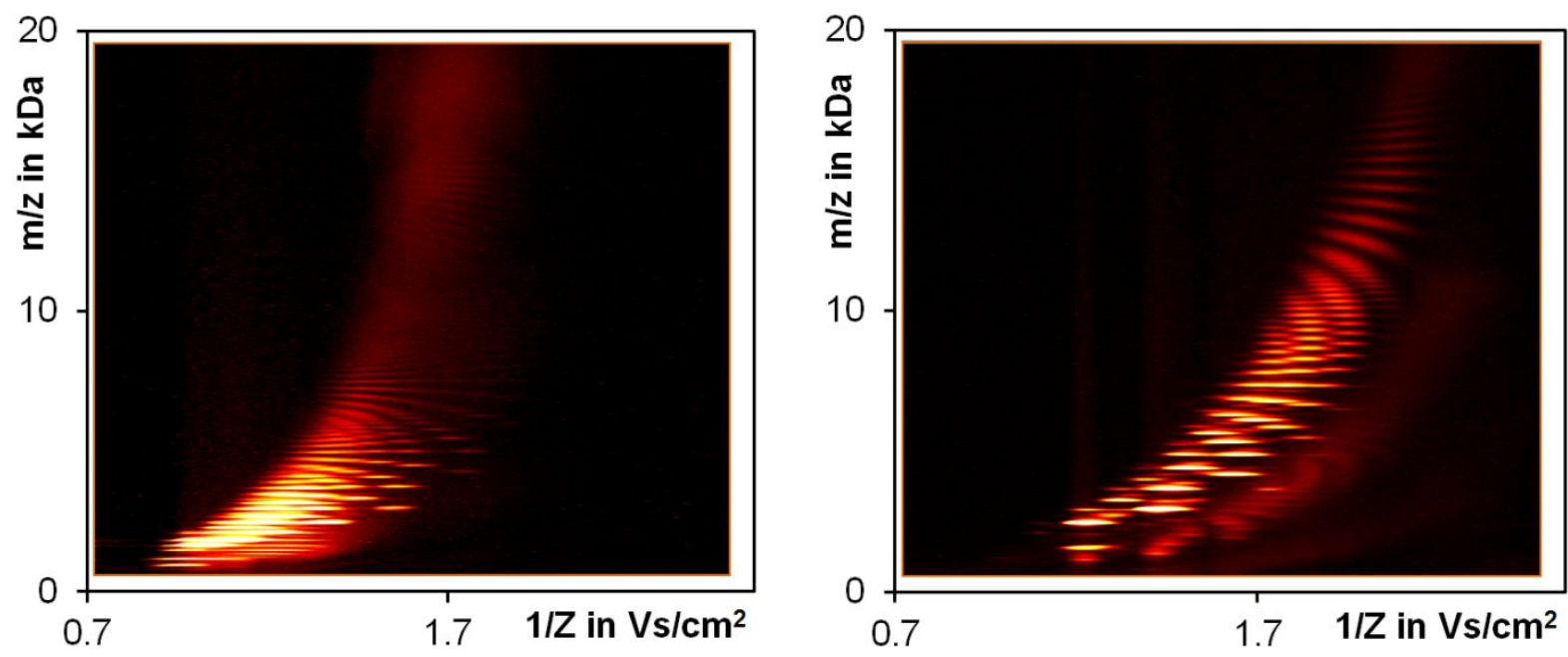

Figure sla: Mobility-classified mass spectra at a concentration of $101 \mu \mathrm{mol}$ per liter. Left: positive ion mode, right: Negative ion mode
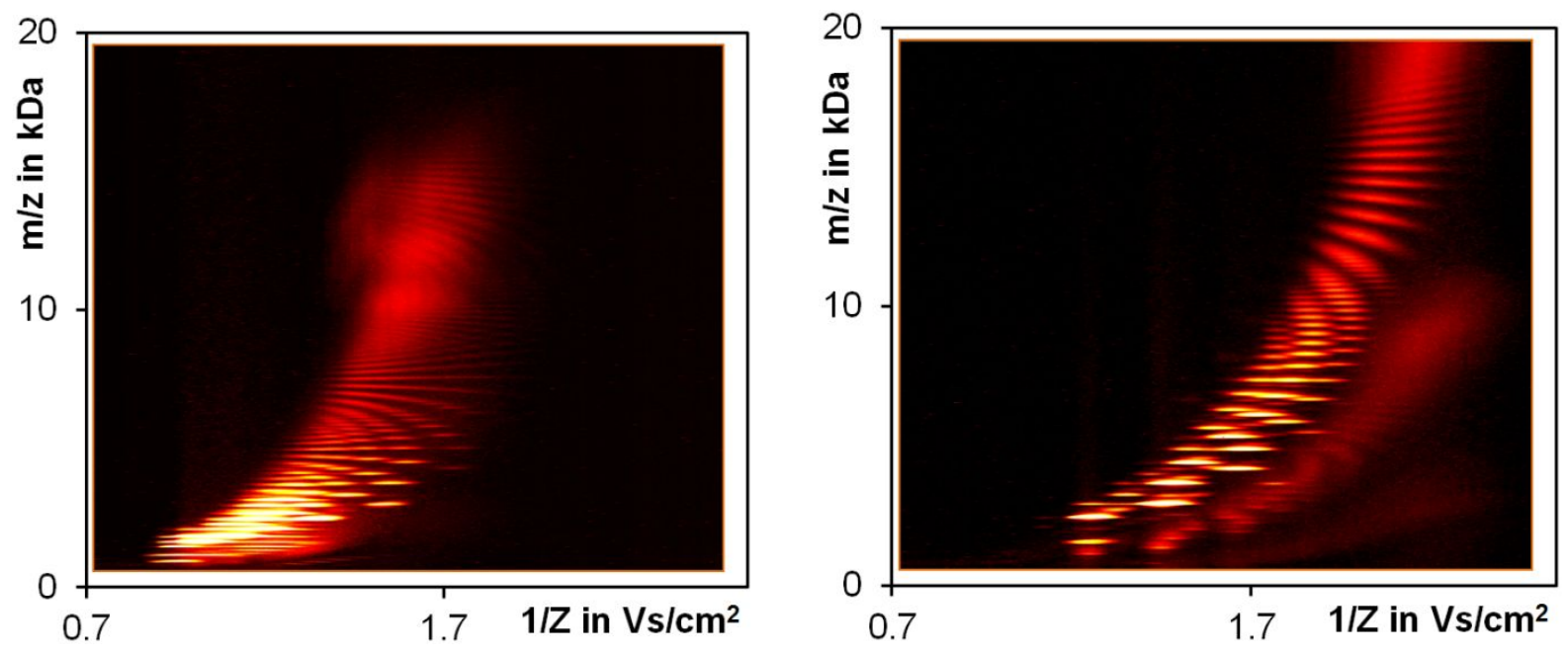

Figure s 1b: Mobility-classified mass spectra at a concentration of $51 \mu$ mol per liter. Left: positive ion mode, right: Negative ion mode 

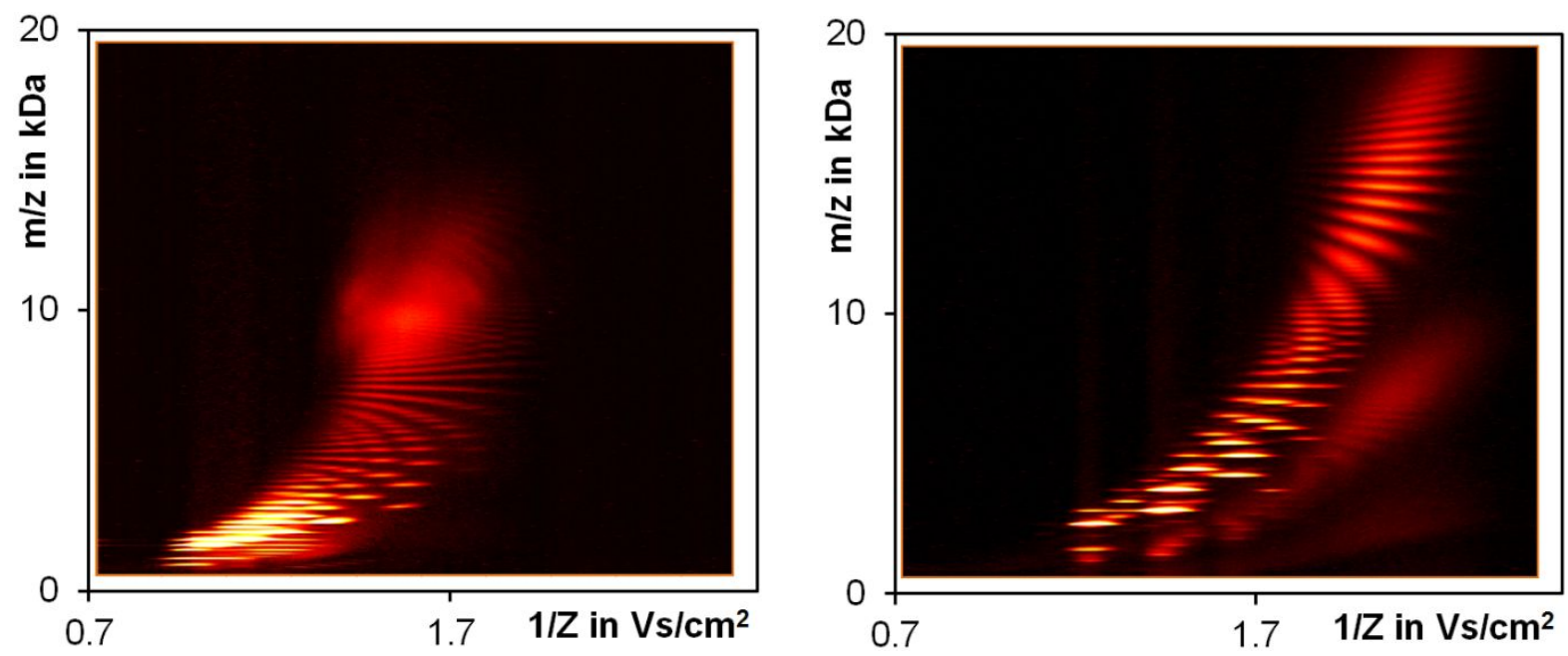

Figure s1c: Mobility-classified mass spectra at a concentration of $34 \mu \mathrm{mol}$ per liter. Left: positive ion mode, right: Negative ion mode
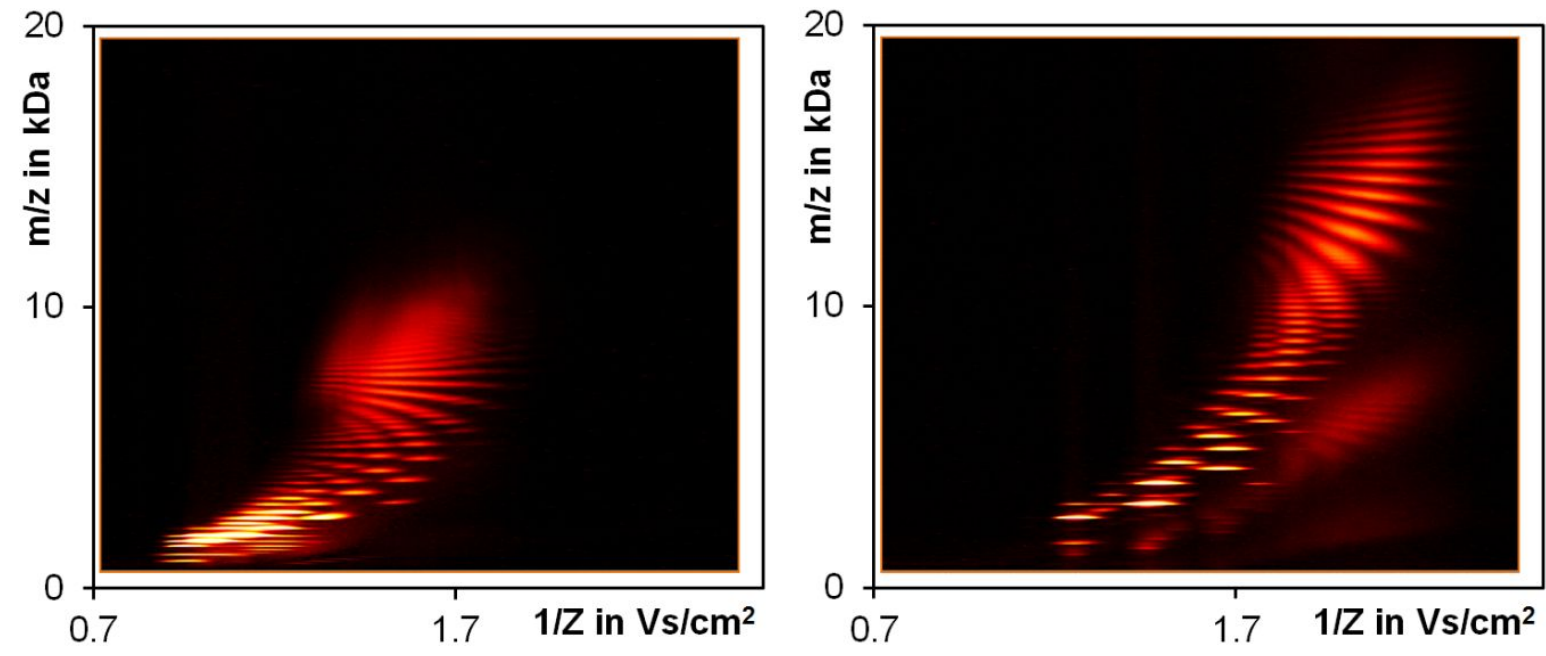

Figure s1d: Mobility-classified mass spectra at a concentration of $23 \mu \mathrm{mol}$ per liter. Left: positive ion mode, right: Negative ion mode 

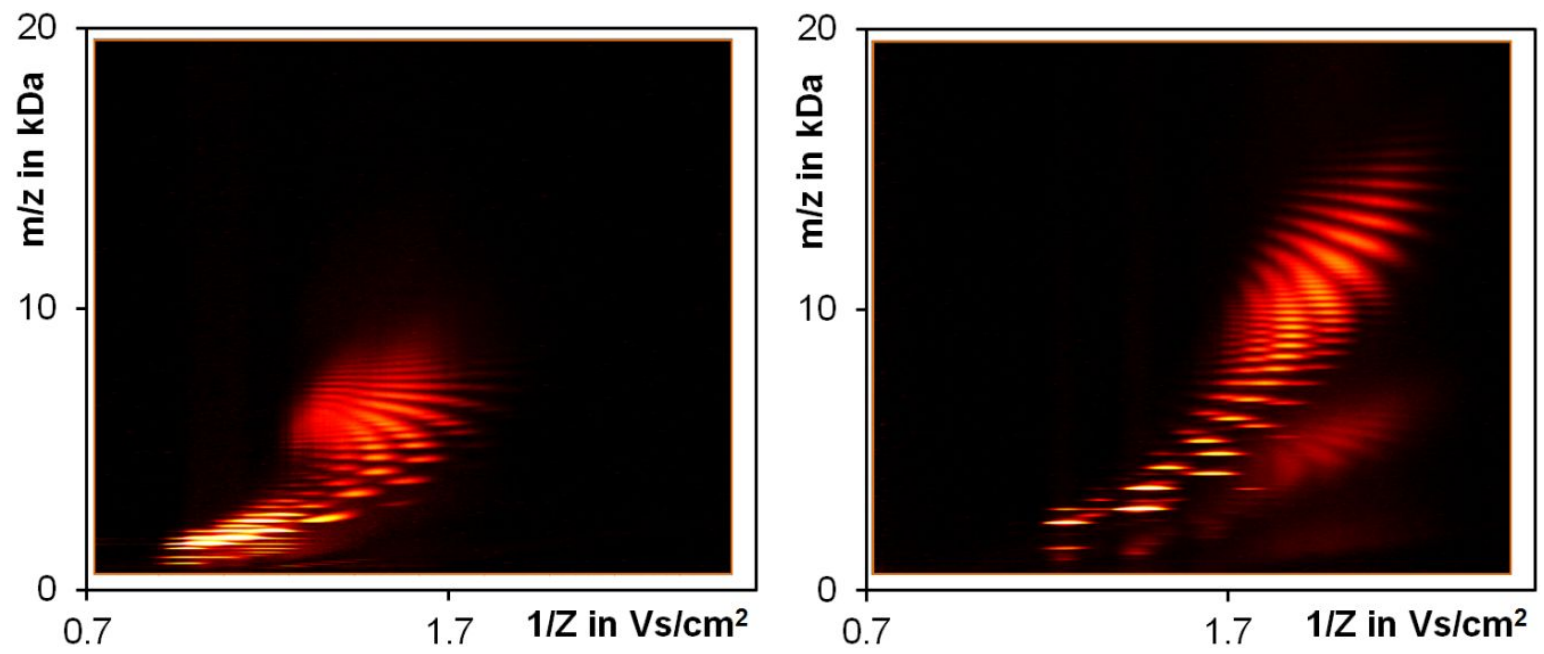

Figure sle: Mobility-classified mass spectra at a concentration of $15 \mu \mathrm{mol}$ per liter. Left: positive ion mode, right: Negative ion mode
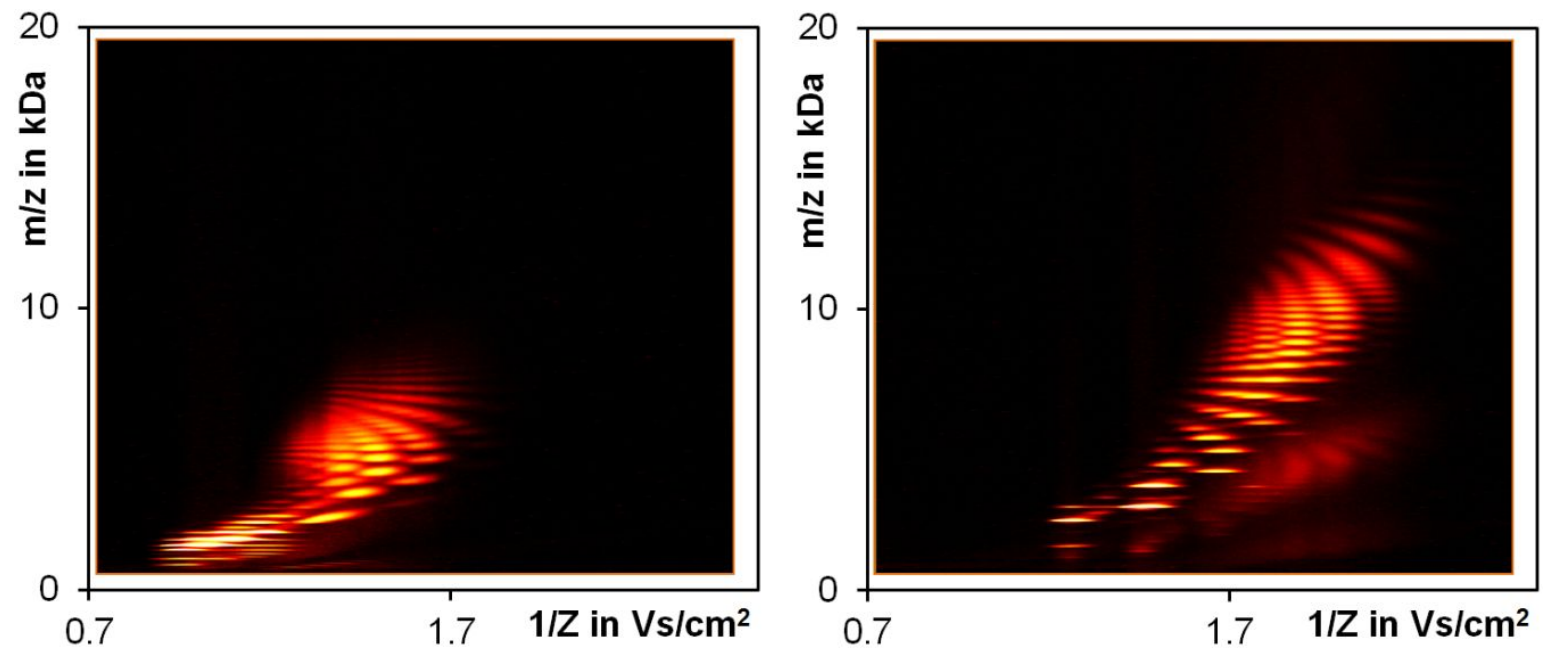

Figure s1f: Mobility-classified mass spectra at a concentration of 10umol per liter. Left: positive ion mode, right: Negative ion mode 

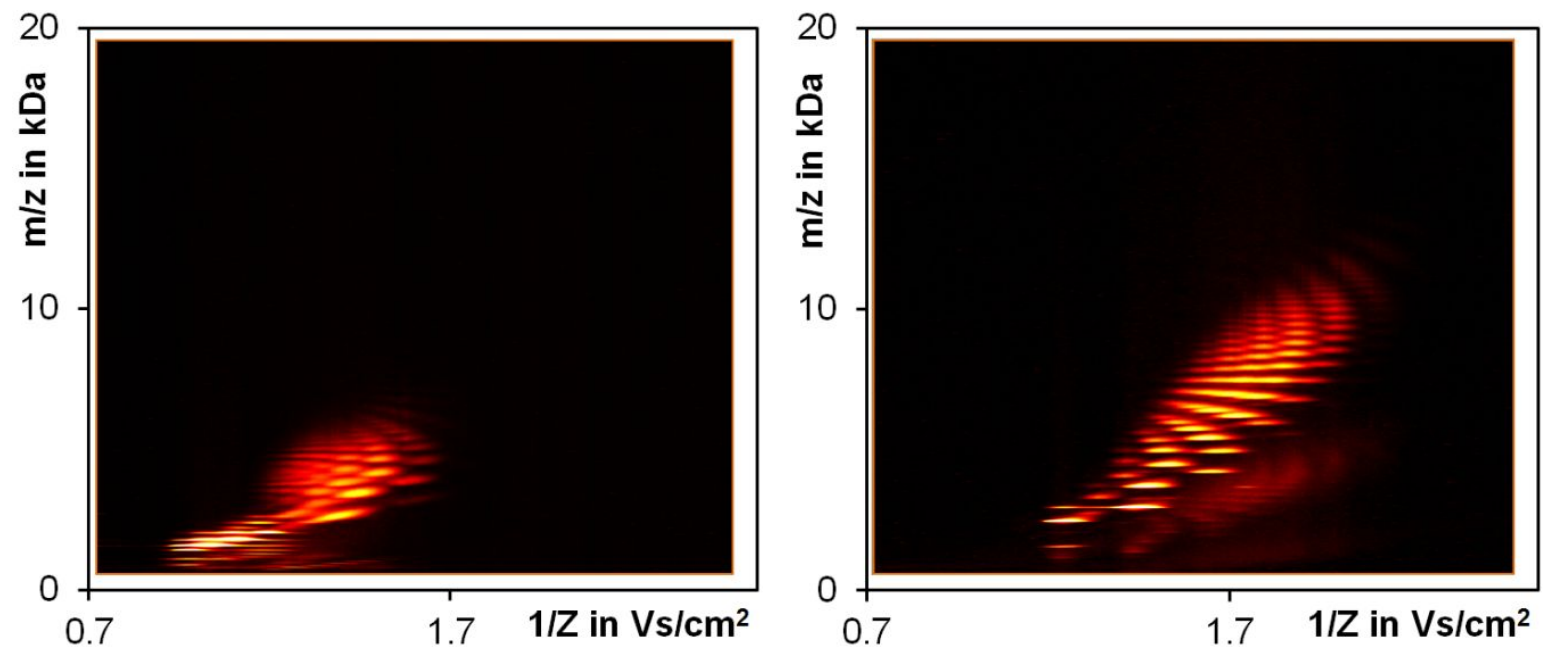

Figure s Ig: Mobility-classified mass spectra at a concentration of $6.7 \mu \mathrm{mol}$ per liter. Left: positive ion mode, right: Negative ion mode
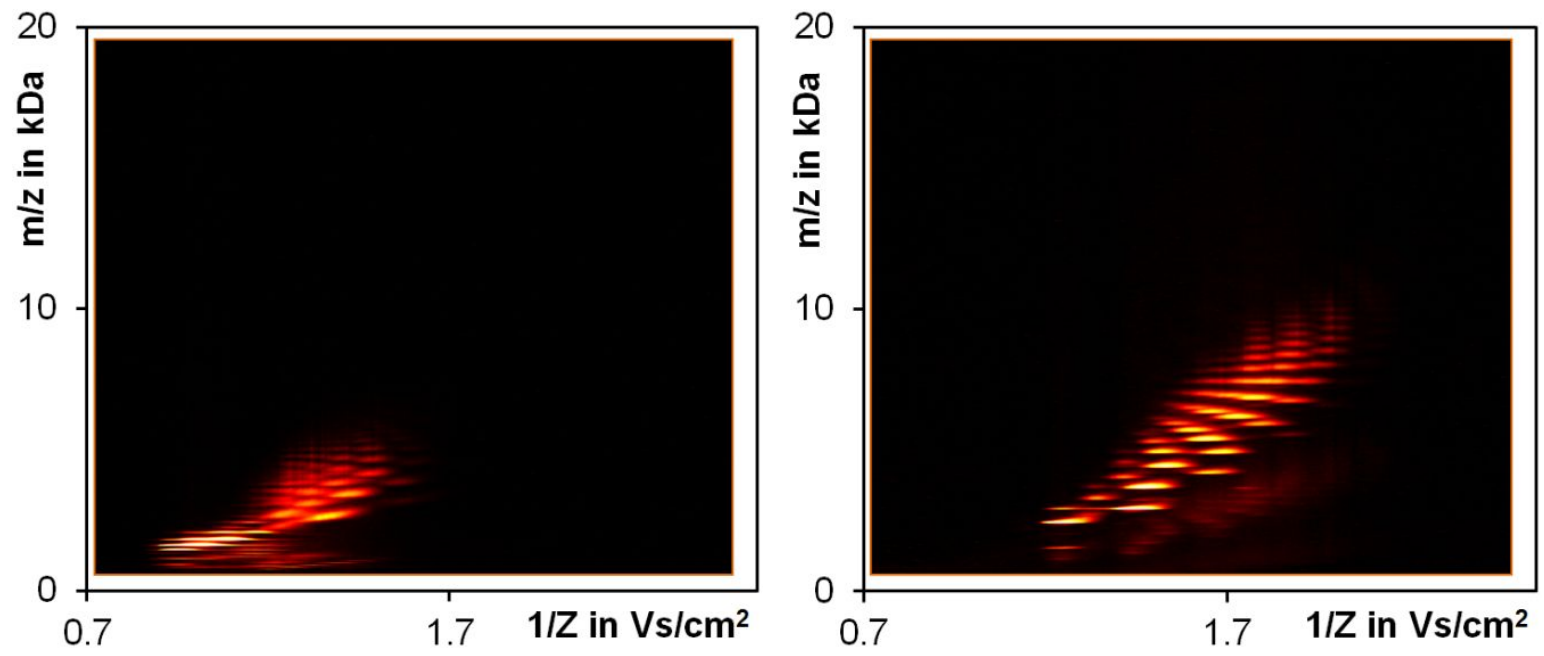

Figure s 1h: Mobility-classified mass spectra at a concentration of 4.5 $\mu$ mol per liter. Left: positive ion mode, right: Negative ion mode 

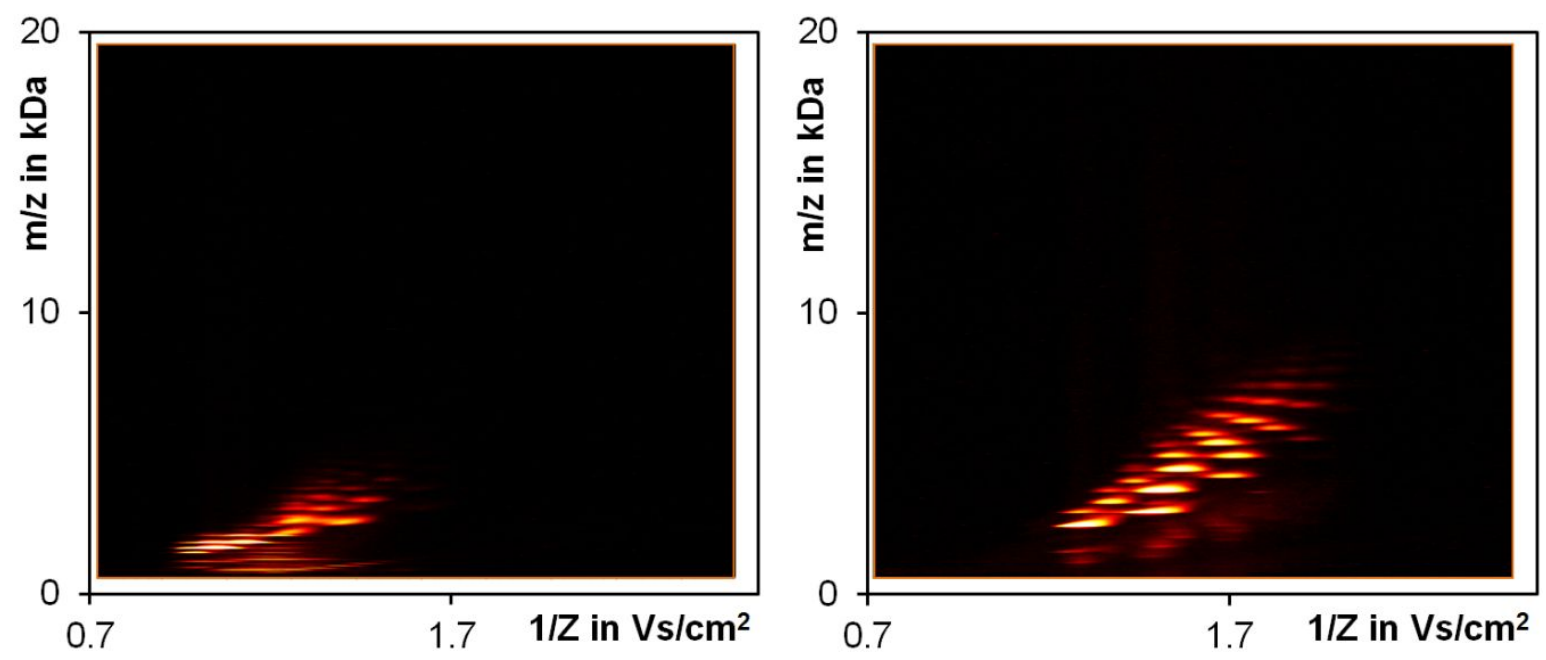

Figure s1i: Mobility-classified mass spectra at a concentration of $2.2 \mu m o l$ per liter. Left: positive ion mode, right: Negative ion mode
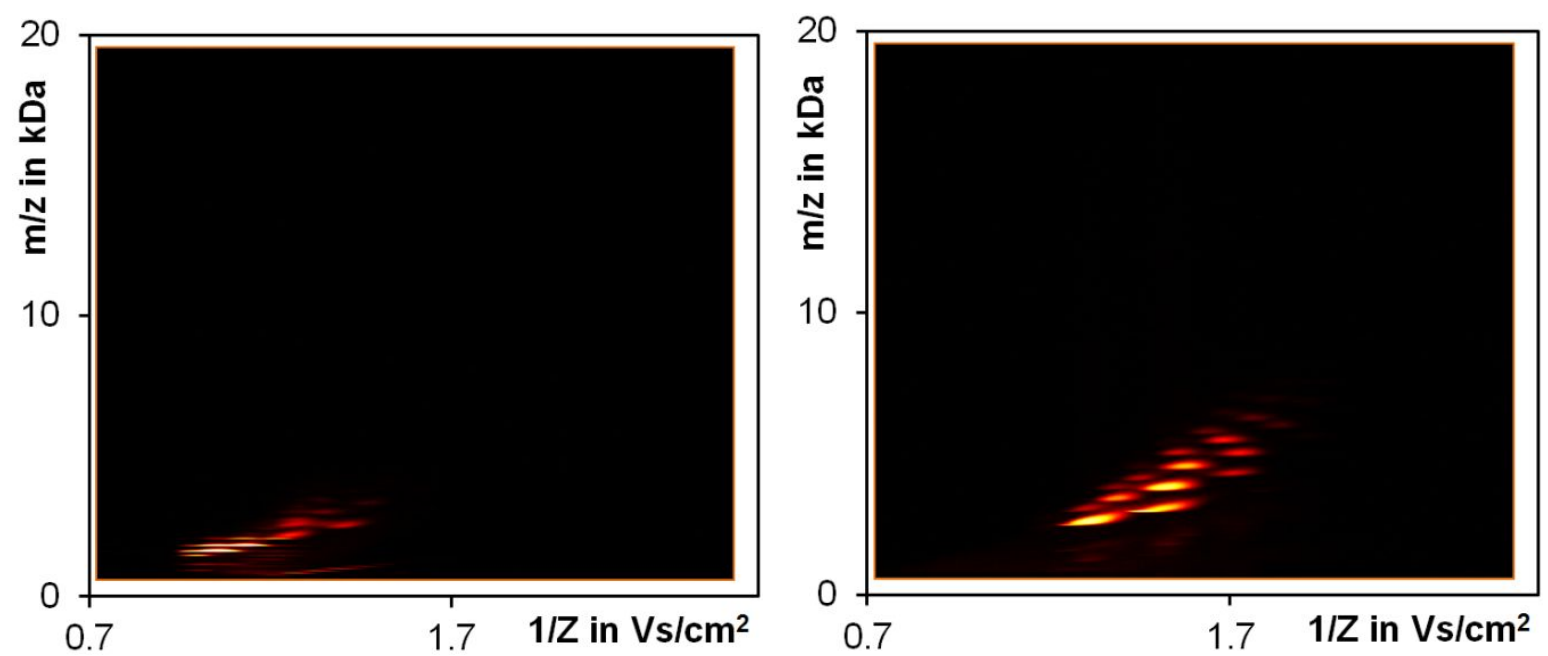

Figure s1j: Mobility-classified mass spectra at a concentration of $1.1 \mu$ mol per liter. Left: positive ion mode, right: Negative ion mode 

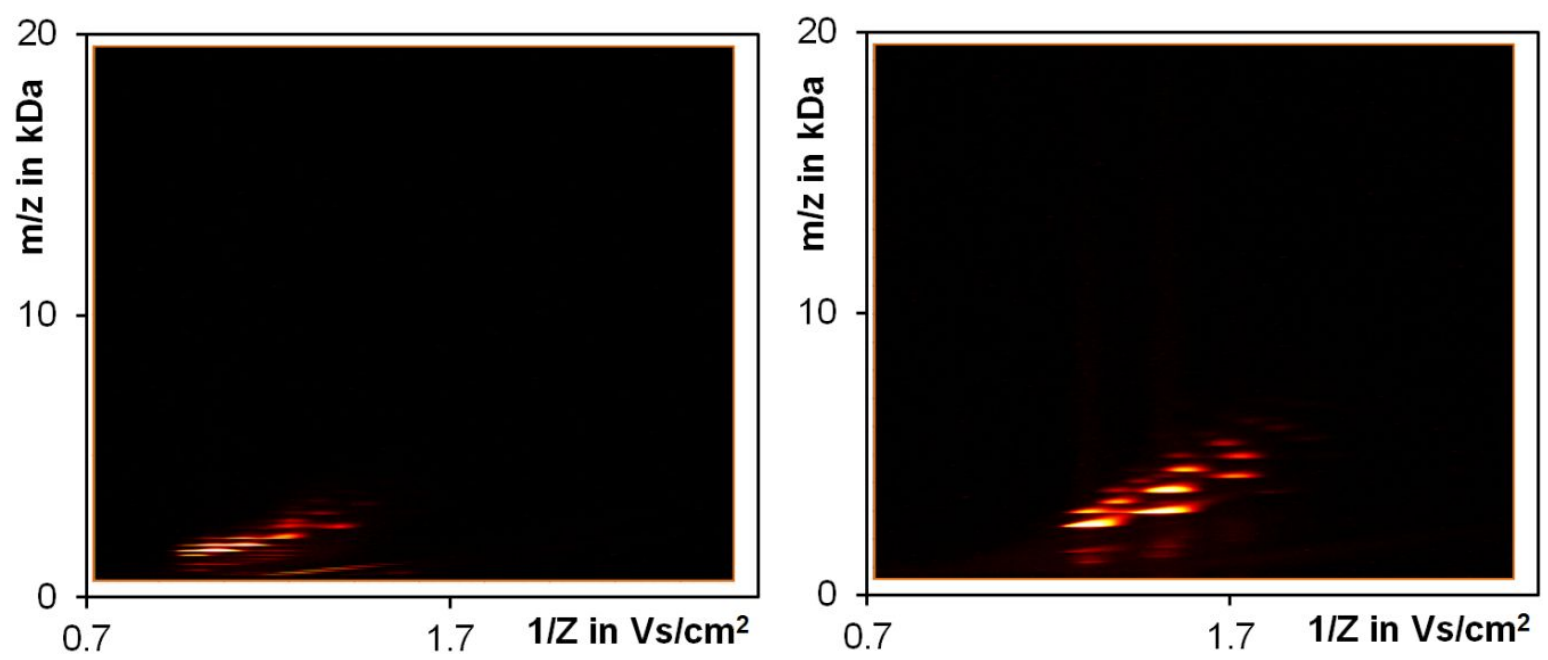

Figure s1k: Mobility-classified mass spectra at a concentration of $0.5 \mu \mathrm{mol}$ per liter. Left: positive ion mode, right: Negative ion mode
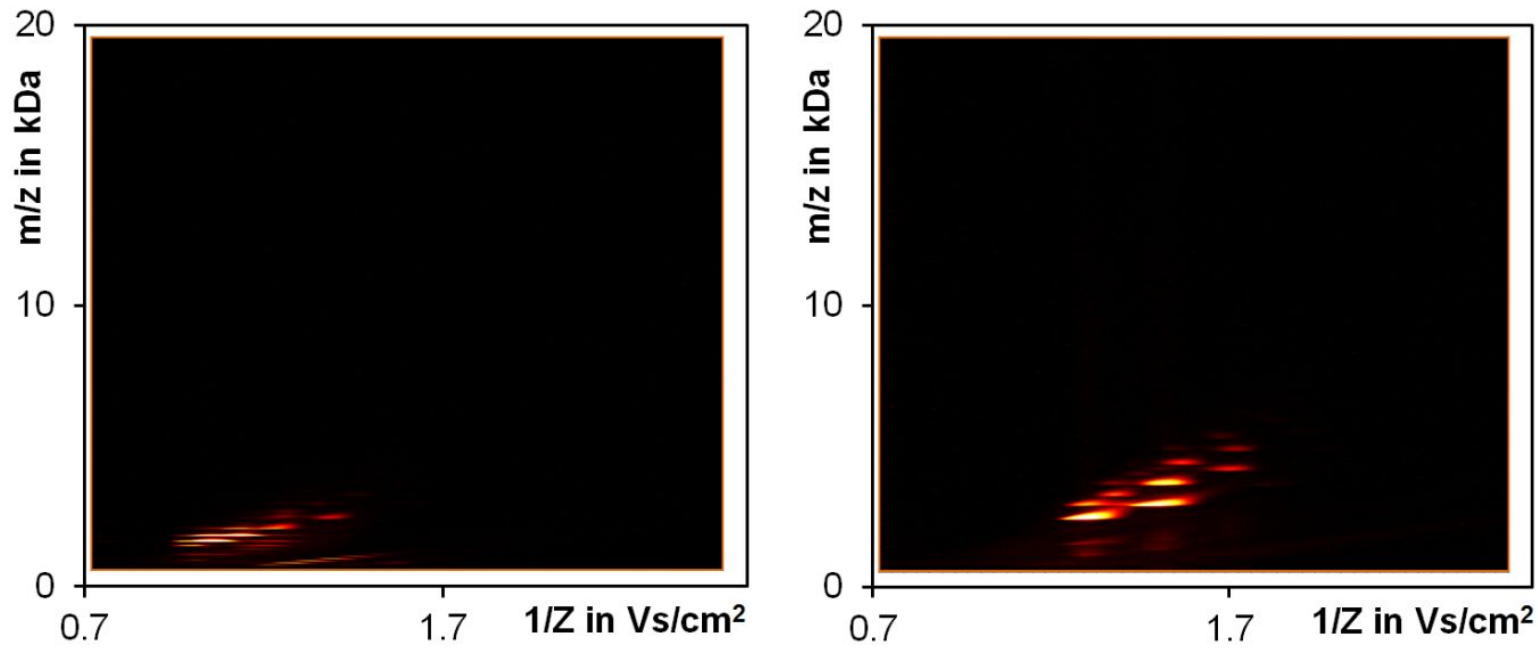

Figure s1l: Mobility-classified mass spectra at a concentration of 0.28umol per liter. Left:

Positive ion mode, right: Negative ion mode

\section{$\underline{\text { A comment on color maps }}$}

Due to the large data size of a mobility-classified mass spectrum (around 300Mb per scan) data import to MatLab® is time quite consuming. The above figures (fig. s1) are images exported from the MS-software. For comparison to the images as provided in the manuscript we show 
pictures of the $23 \mu \mathrm{mol} / 1$ (negative) in the following two images (figure s2). The left figure is the same as in the manuscript (without model match), the right picture shows the same data if the color map covers the full intensity scale.
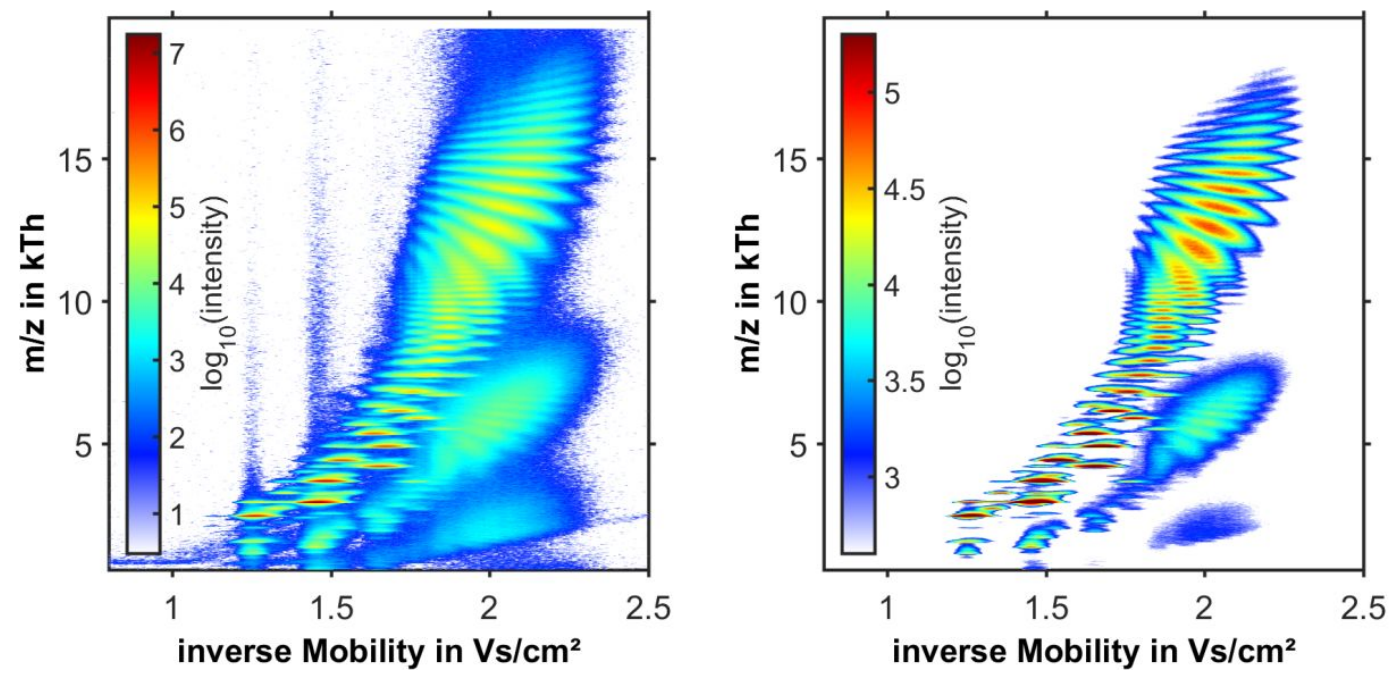

Figure s2: Same data as in figure s1c (right). Left: Color map scaled for the full intensity range.

Right: Color map scaled for a limited intensity range as in the manuscript, to allow for better judgement of parent droplets cluster mode. 

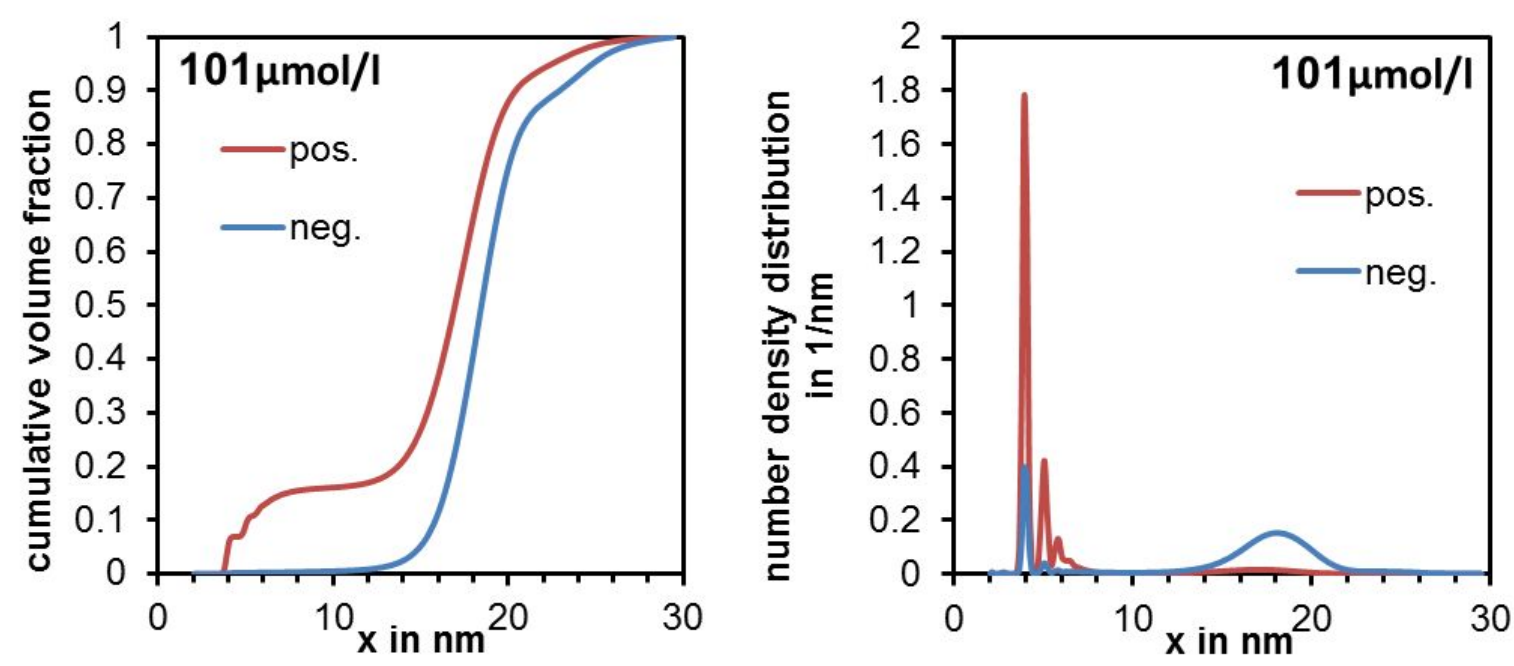

Figure s3a: Size spectra measured by SMPS in positive and negative spray polarity at a concentration of $101 \mu \mathrm{mol}$ per liter. Left: Cumulative volume distribution, right: number density distribution.
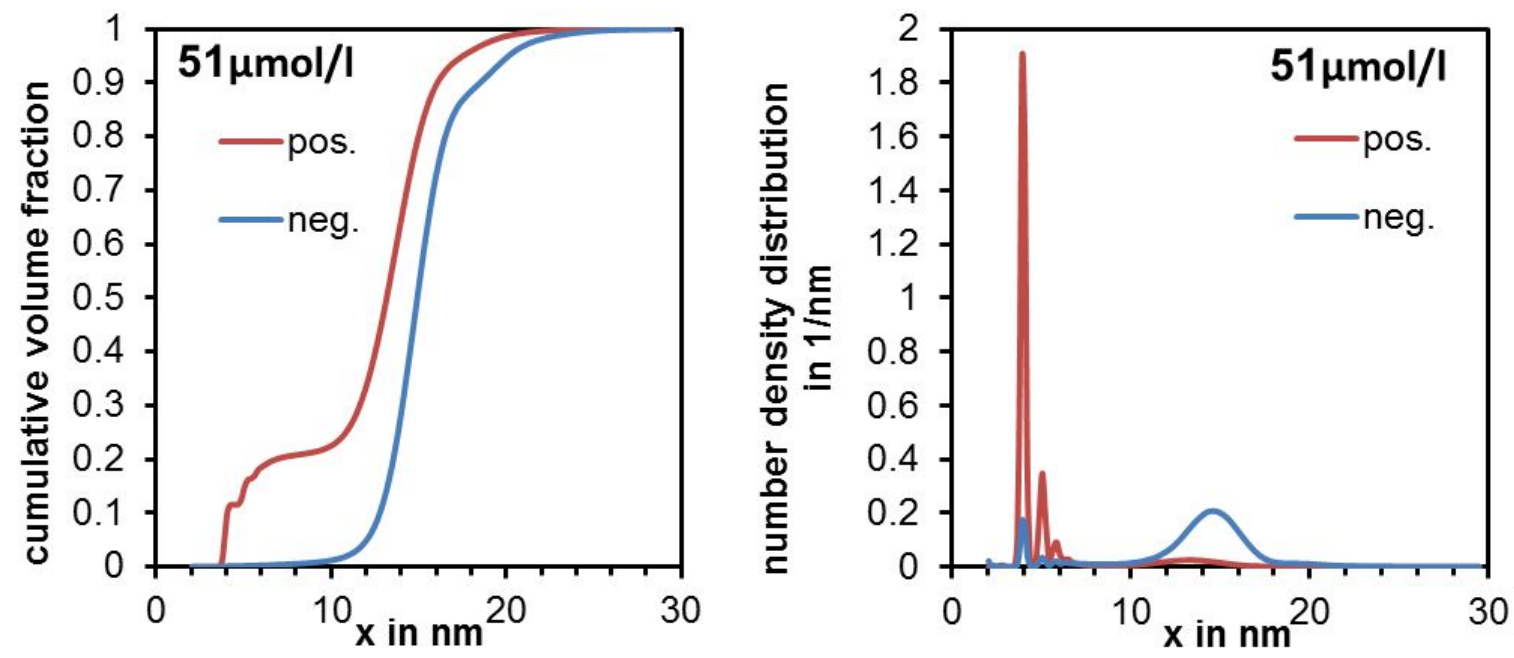

Figure s3b: Size spectra measured by SMPS in positive and negative spray polarity at a concentration of $51 \mu \mathrm{mol}$ per liter. Left: Cumulative volume distribution, right: Number density distribution. 

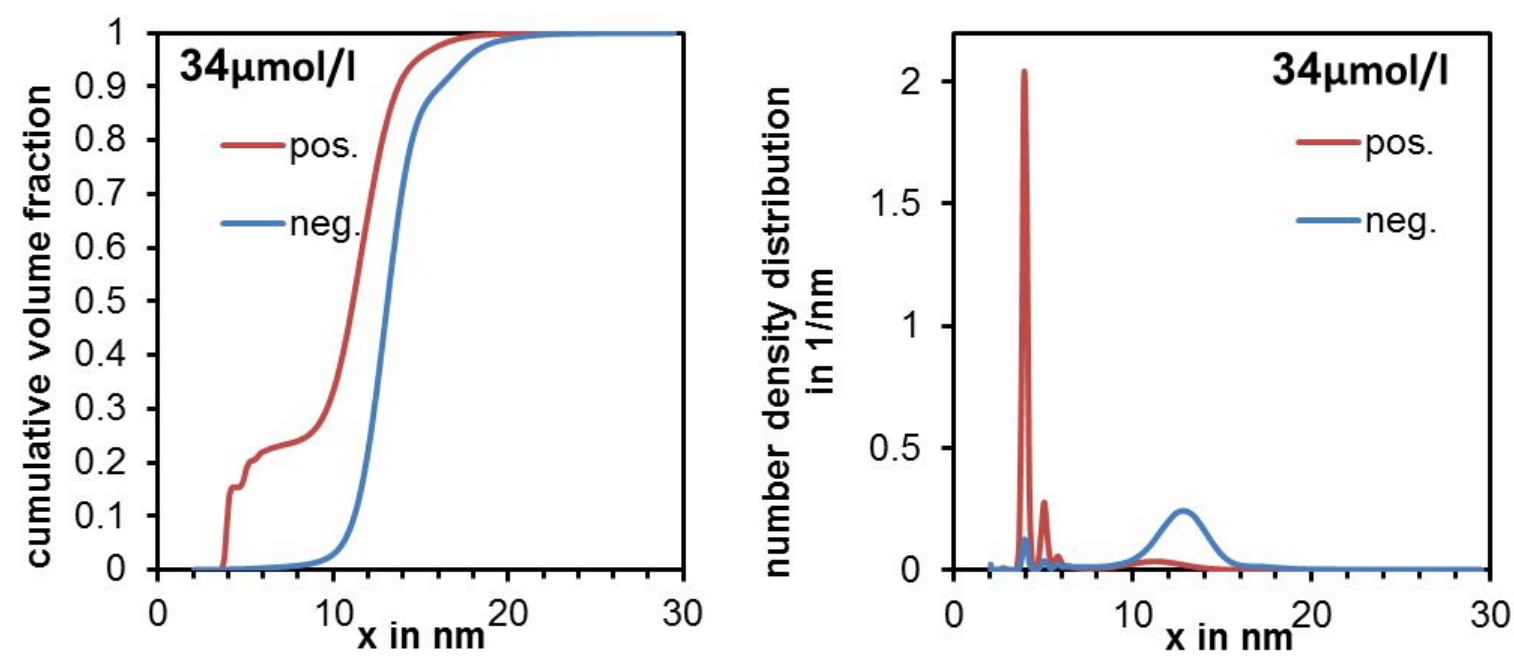

Figure s3c: Size spectra measured by SMPS in positive and negative spray polarity at a concentration of $34 \mu \mathrm{mol}$ per liter. Left: Cumulative volume distribution, right: Number density distribution.
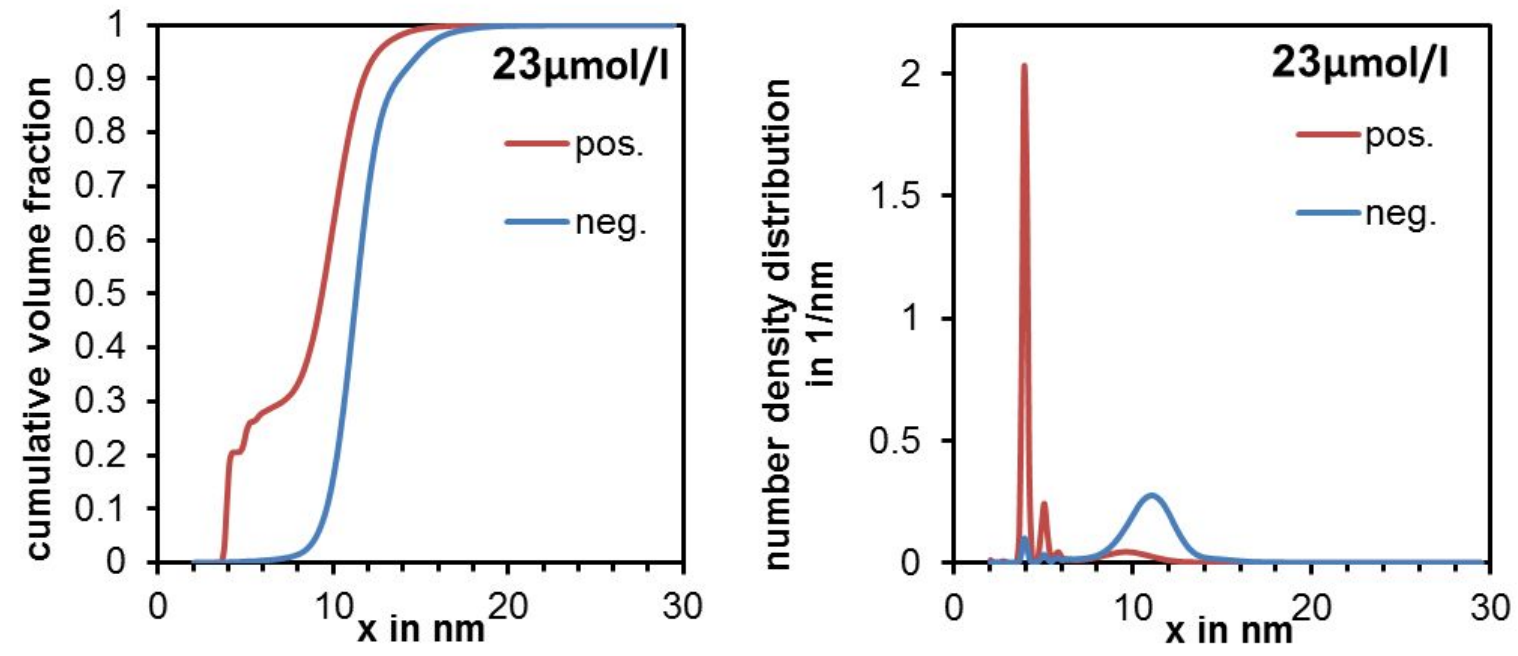

Figure s3d: Size spectra measured by SMPS in positive and negative spray polarity at a concentration of $23 \mu \mathrm{mol}$ per liter. Left: Cumulative volume distribution, right: Number density distribution. 

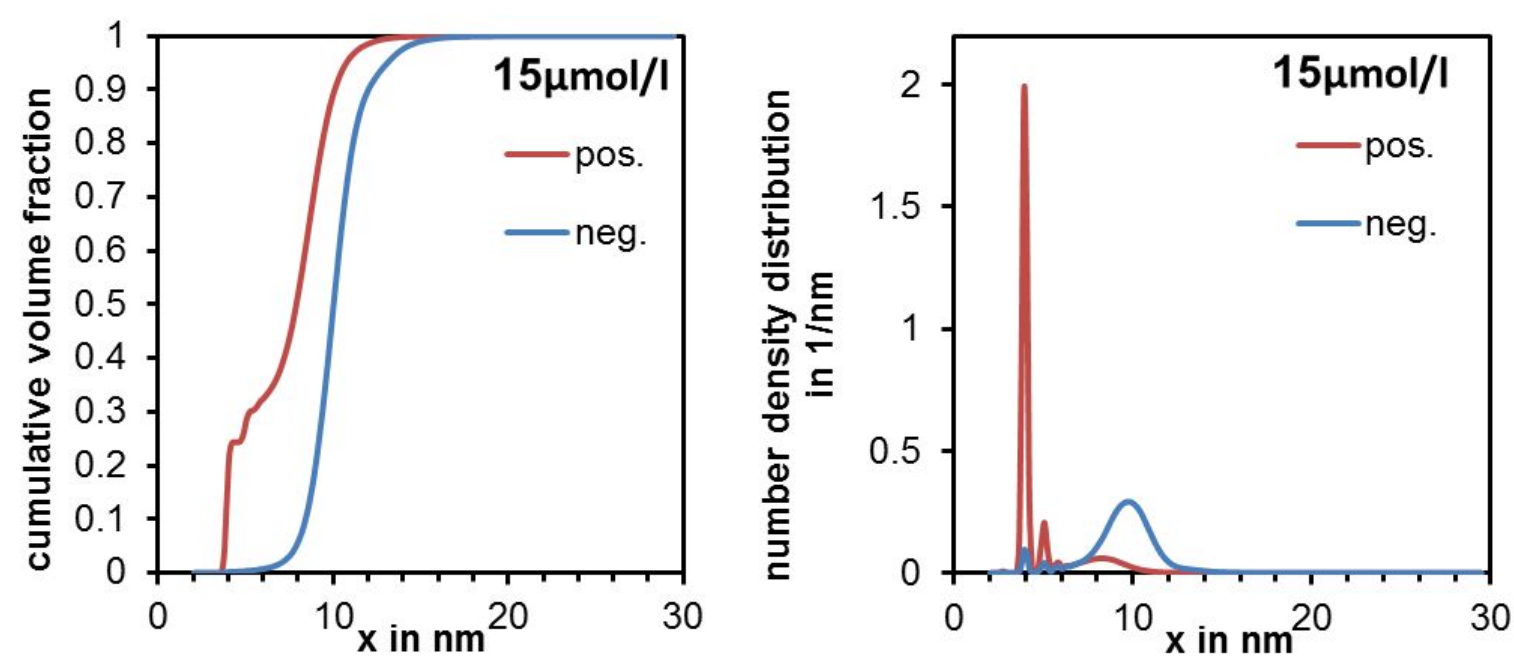

Figure s3e: Size spectra measured by SMPS in positive and negative spray polarity at a concentration of $15 \mu \mathrm{mol}$ per liter. Left: Cumulative volume distribution, right: Number density distribution.
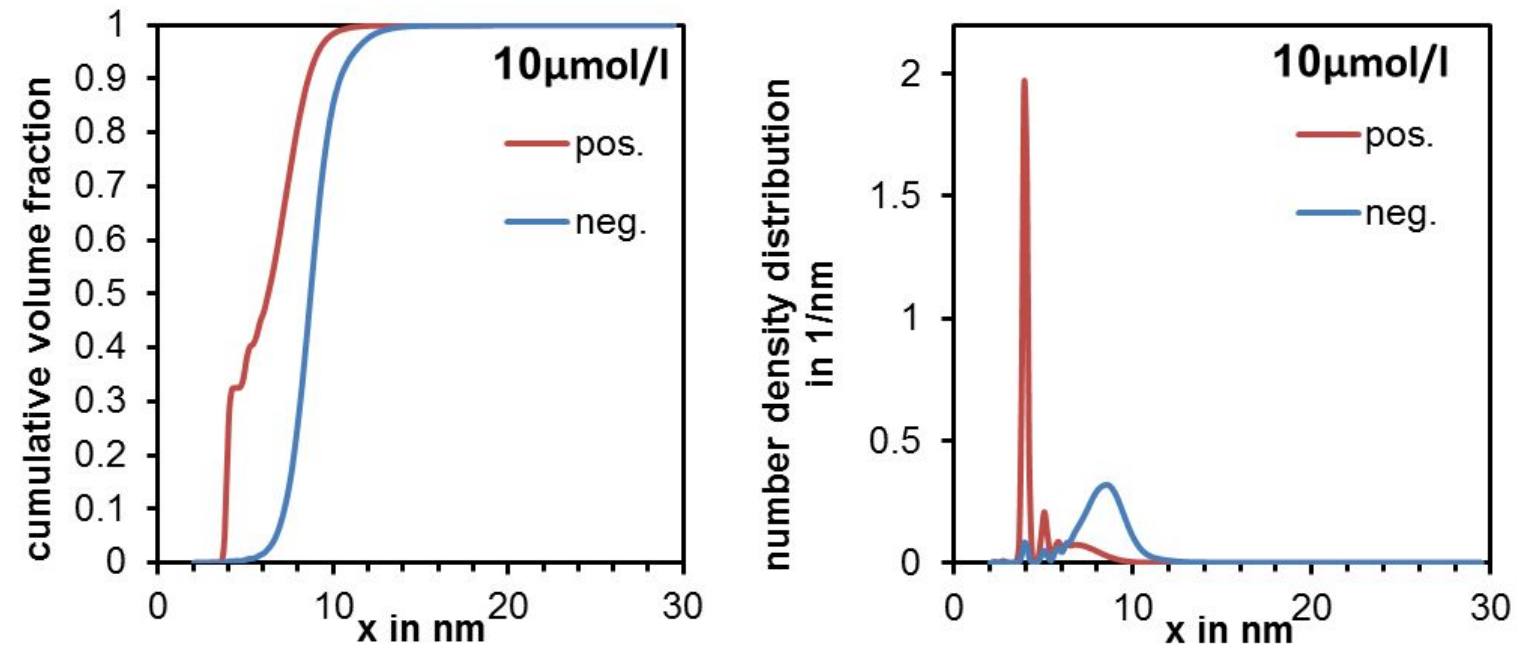

Figure s3f: Size spectra measured by SMPS in positive and negative spray polarity at a concentration of $10 \mu m o l$ per liter. Left: Cumulative volume distribution, right: Number density distribution. 

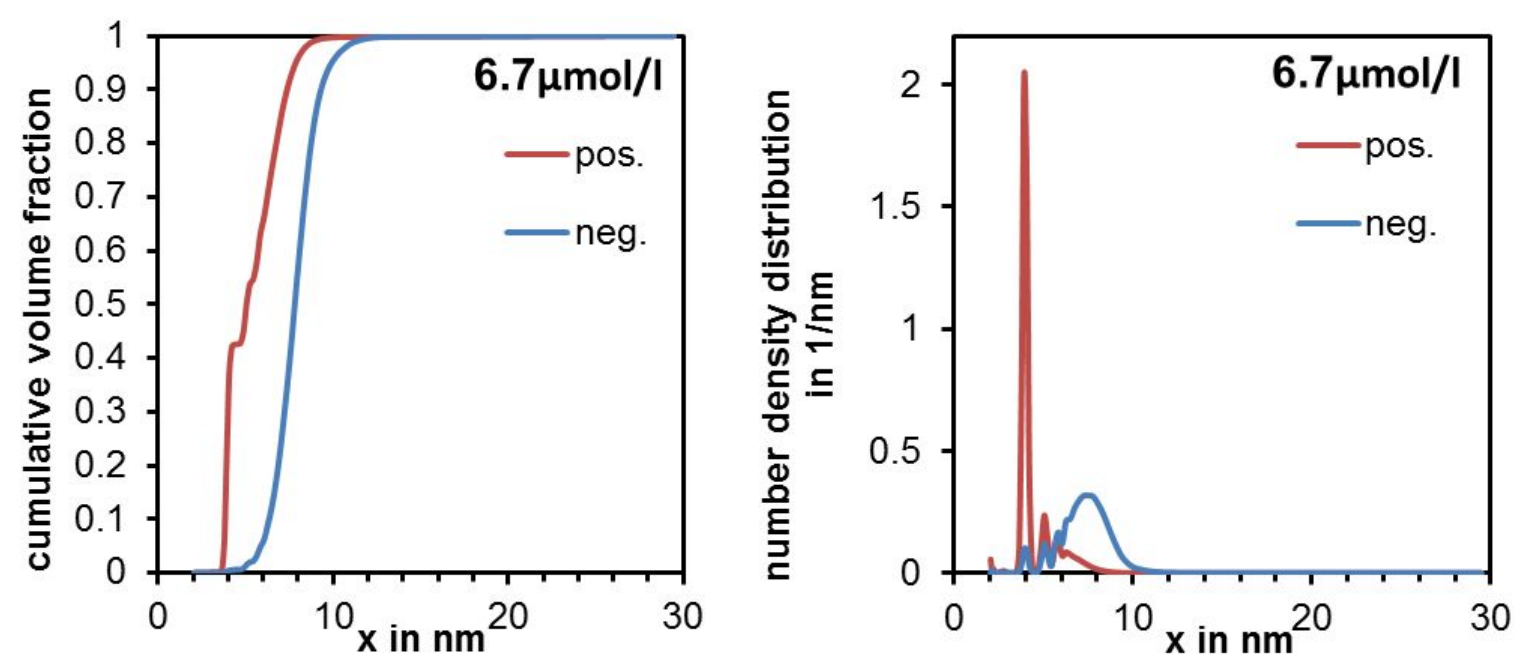

Figure s3g: Size spectra measured by SMPS in positive and negative spray polarity at a concentration of $6.7 \mu \mathrm{mol}$ per liter. Left: Cumulative volume distribution, right: Number density distribution.
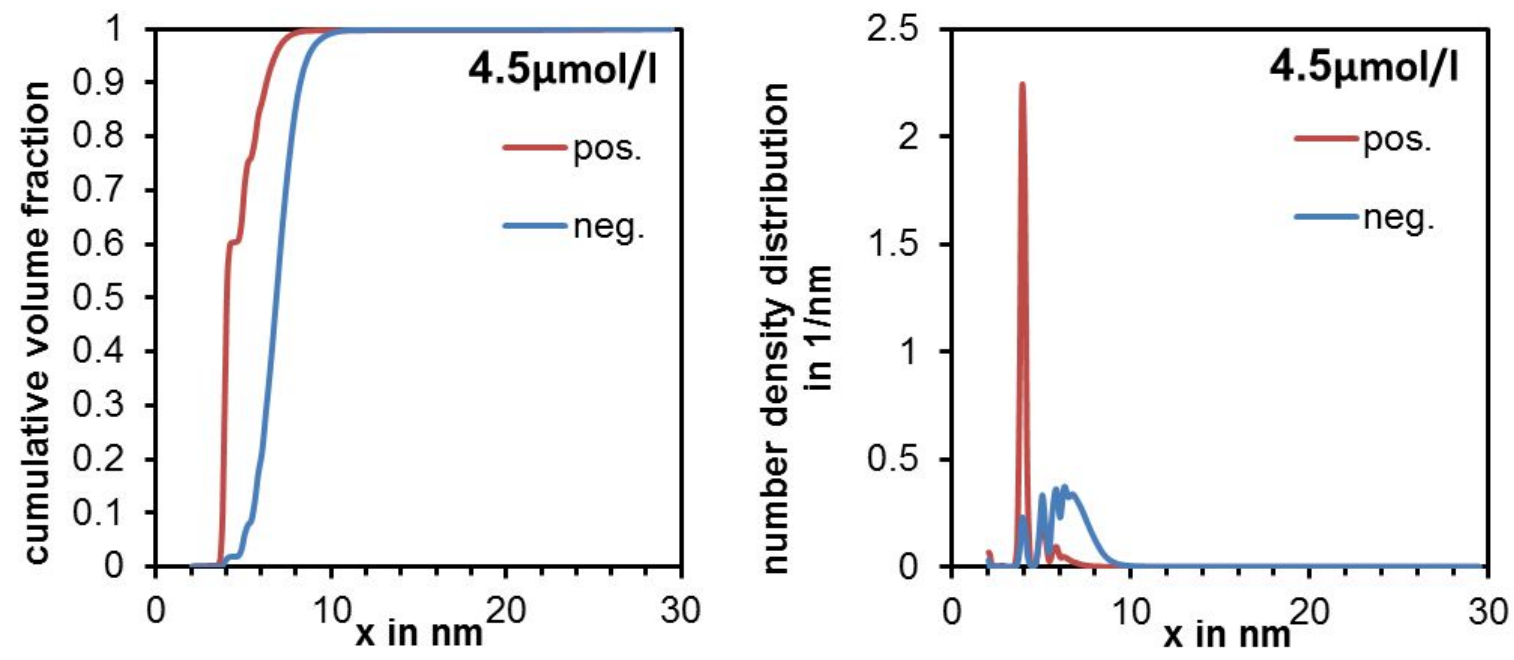

Figure s3h: Size spectra measured by SMPS in positive and negative spray polarity at a concentration of $4.5 \mu \mathrm{mol}$ per liter. Left: Cumulative volume distribution, right: Number density distribution. 

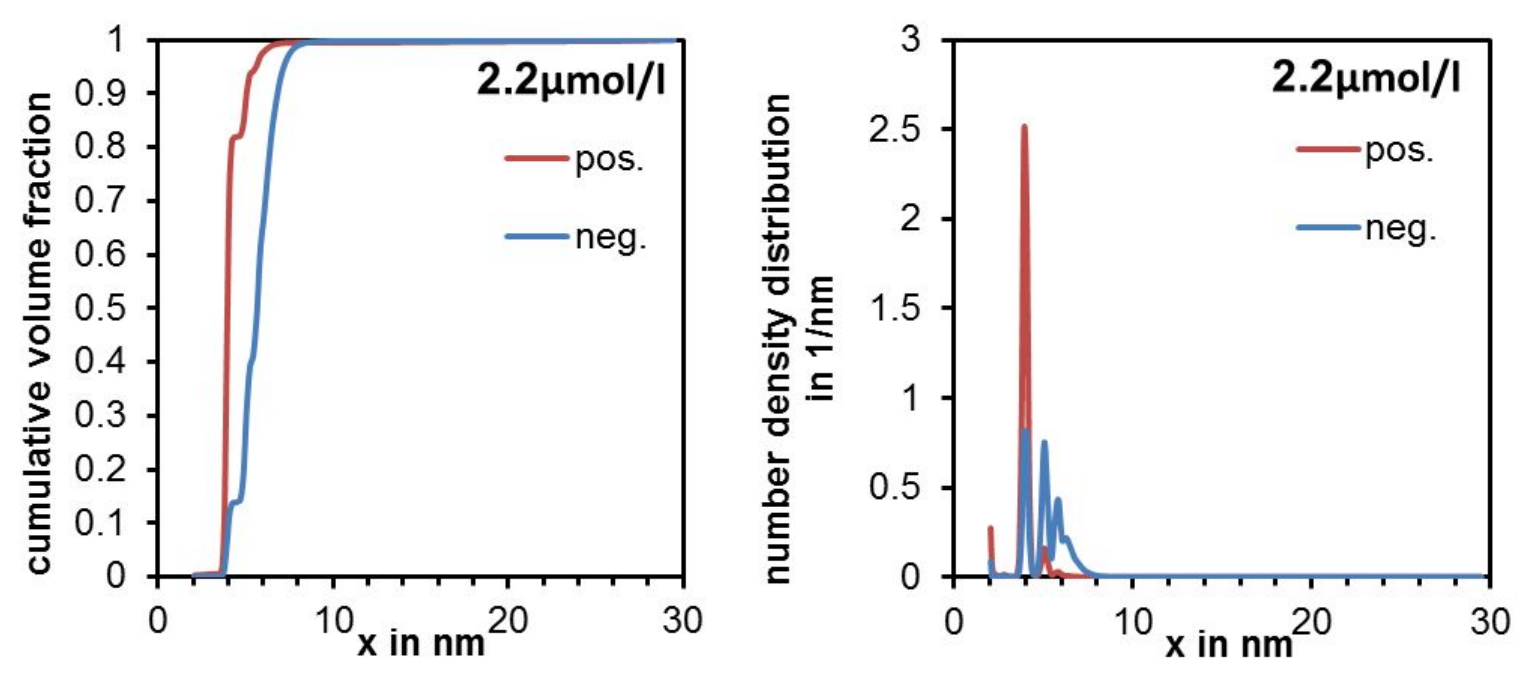

Figure s3i: Size spectra measured by SMPS in positive and negative spray polarity at a concentration of $2.2 \mu \mathrm{mol}$ per liter. Left: Cumulative volume distribution, right: Number density distribution.
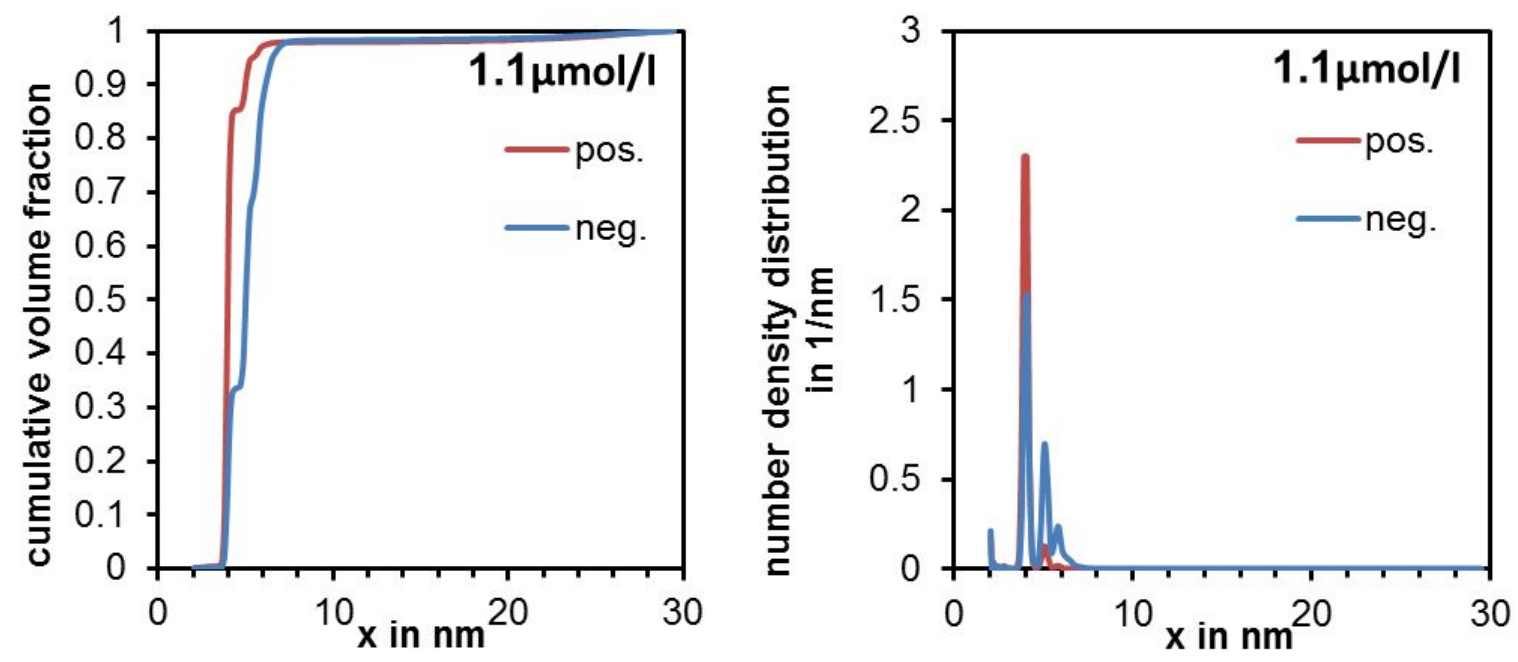

Figure s3j: Size spectra measured by SMPS in positive and negative spray polarity at a concentration of 1.1 mol per liter. Left: Cumulative volume distribution, right: Number density distribution. 

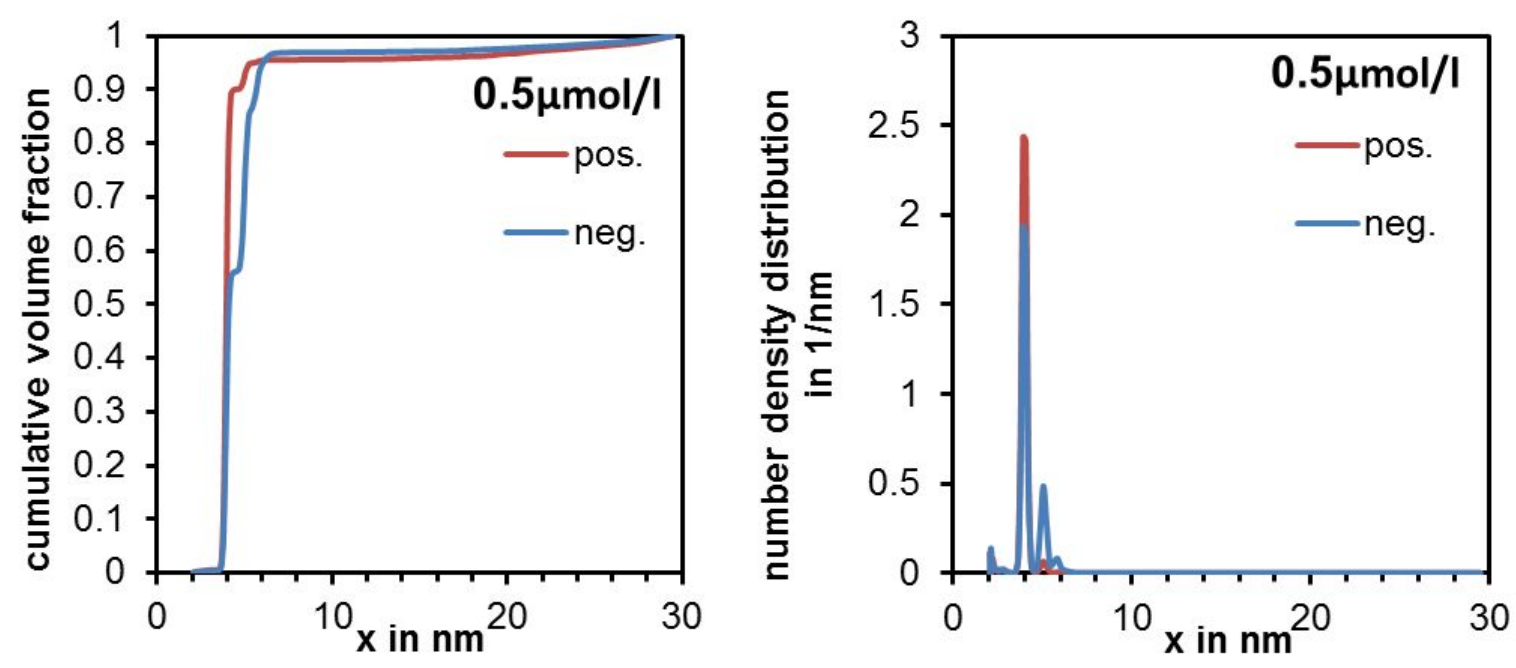

Figure s3k: Size spectra measured by SMPS in positive and negative spray polarity at a concentration of $0.5 \mu \mathrm{mol}$ per liter. Left: Cumulative volume distribution, right: Number density distribution.
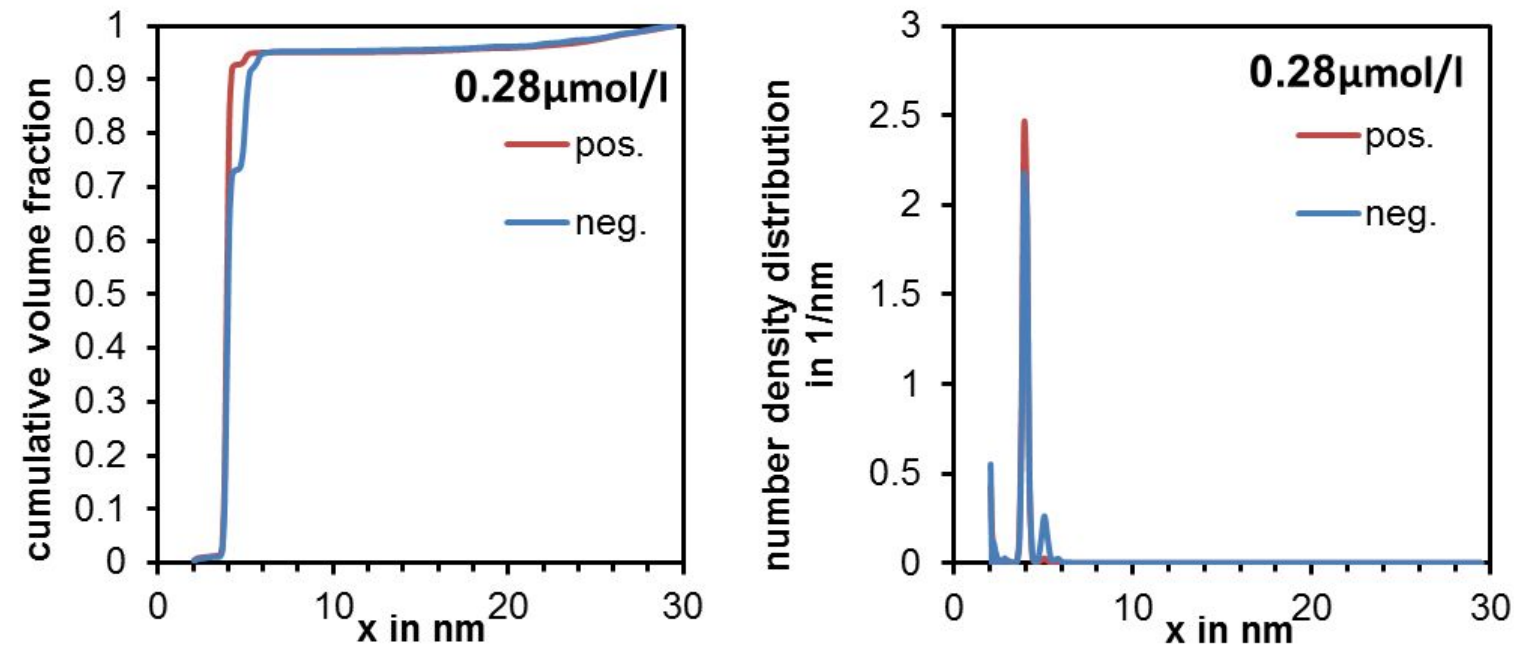

Figure s3l: Size spectra measured by SMPS in positive and negative spray polarity at a concentration of $0.28 \mu \mathrm{mol}$ per liter. Left: Cumulative volume distribution, right: Number density distribution. 
A comment on extraction of size distribution from DMA-MS spectra and comparison to SMPS

$\underline{\text { data }}$

In the manuscript we compare parent droplet cluster mode numbers of proteins as derived from SMPS size spectra with data from mobility-classified mass spectrometry by indicating the calculated location of those cluster ions in the DMA MS-scan.
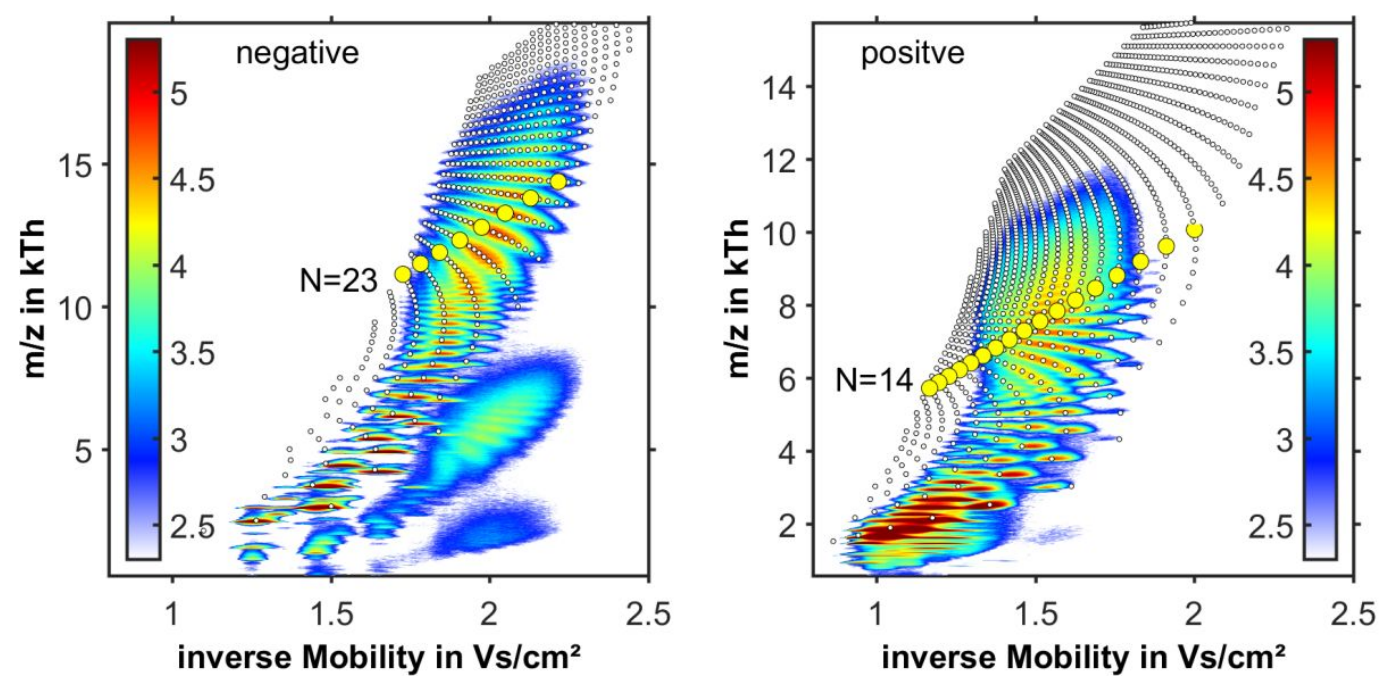

Figure s4: Same figure as figure 8 (middle row) in the manuscript.

Practically, the signal intensity as function of the particle diameter can easily be extracted from the scans once they are imported to MatLab ${ }^{\circledR}$, a complete distribution can be derived as well. The simplest approach to do that is integration of the signal intensity in orthogonal direction to the lines, on which particles with equal size are located. Lines connecting the mode cluster sizes in figure s4 are such lines, for example. Figure s5 shows such lines for clusters of $N=1 \ldots 12$. This very simple approach works nearly perfectly in case that cluster shape and density are identical for all clusters and the cluster centers are well-matched by the model. As described in the manuscript the cluster locations are matched using a monomer mass that applies to the (partly solvated) monomer mass in relatively large clusters. The match of the location of a certain cluster 
size for the parent droplets is thus relatively good. (Note: Any deviation from the assumed constant shape and constant density condition results with this very simple model in a data misinterpretation. For example, a cluster, which has a lower density and is therefore - at given mass and assumed fixed shape-larger, is practically located at a higher inverse mobility. A shift to a higher mobility in figure s5 brings this cluster closer to the next smaller cluster location.) If the modeled constant particle size match the practically observed locations the error of this simple approach is very small due to the near Gaussian shape of the mobility peaks. From the integration one obtains the cumulative detected signal intensity as function of particle size. Derivation of this cumulative signal intensity function with respect to particle size results in the signal intensity distribution as function of particle size. If one assumes in lack of better knowledge that each count represents a single particle (detection efficiency is independent of cluster mass, charge state and projected area) one may call the result a particle size distribution.

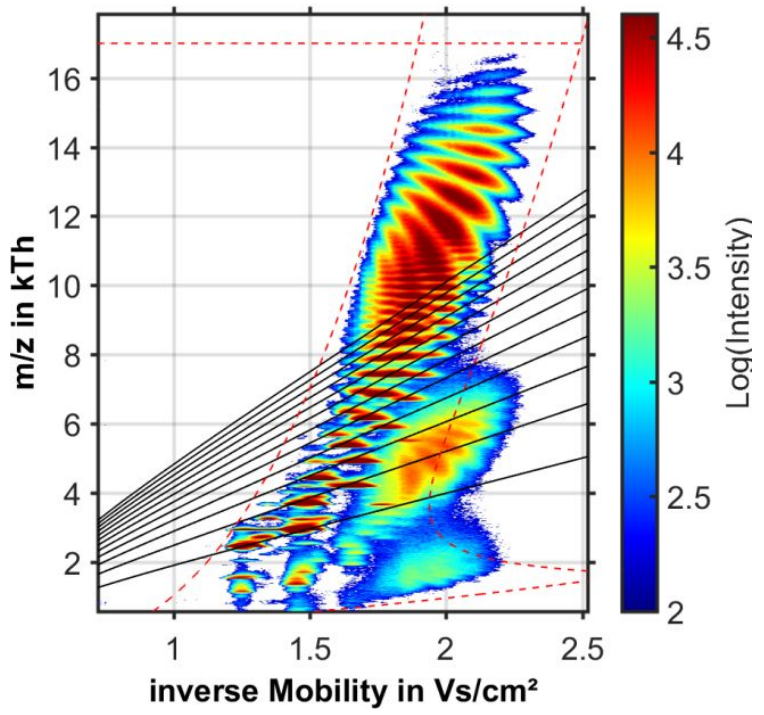

Figure s5: Exemplary lines of constant particle size assuming constant shape and density of the observed species. Signal integration is performed in orthogonal direction to these lines. 
Such size distributions as derived for the $23 \mu \mathrm{mol} / 1$ sample in the positive and negative ion mode are compared in figure 55 to the size distributions measured by SMPS.
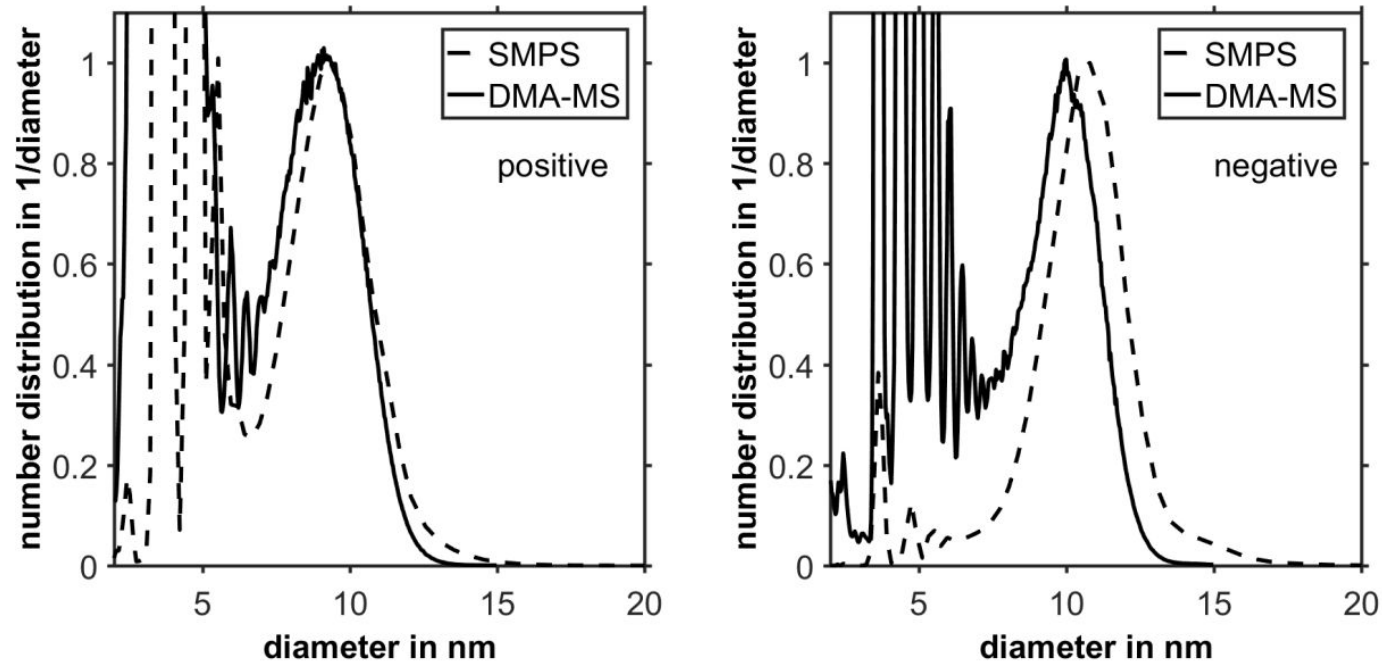

Figure s6: Size distributions as derived from DMA-MS spectra under the assumption of size independent detection efficiency in comparison with spectra measured by SMPS.

The assumption that the cluster detection efficiency is independent of size, mass and charge state is practically naïve. If one e.g. assumes the cluster detection efficiency to be inversely proportional to the cluster mass, one obtains the following match (figure s7) for the same data as in figure s6. While some distribution parameters of the DMA-MS data derived size distribution (such as the mean particle size) change strongly dependent on the detection efficiency assumption, the mode of the parent droplet spectrum is quite robust and thus used as parameter for comparison in the manuscript. 

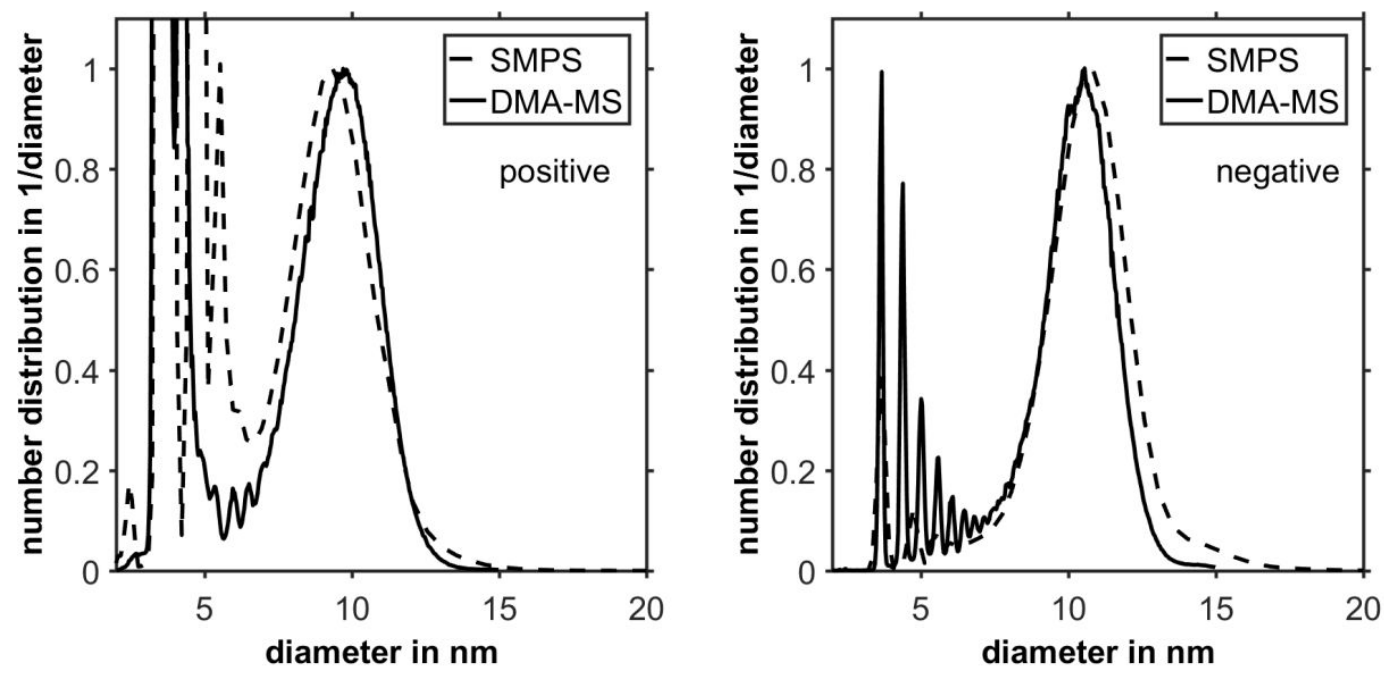

Figure s7: Size distributions as derived from DMA-MS spectra under the (here arbitrary) assumption of a detection efficiency being inversely proportional to the cluster mass in comparison with spectra measured by SMPS.

With respect to the mode location any comparison (fig. s4, fig. s6, fig. s7 ) tells the DMA-MS data are well-represented by SMPS data within limited of uncertainty.

\section{S2: Details on mass spectrometer settings used}

Mass spectrometry of the mobility-classified ions is performed by a Bruker Impct II qQ-

TOF whereby the Seadm-DMA is connected to inlet of the mass spectrometer instead of the original electrospray ion source. To obtain a coherent picture of the classified ions the capillary temperature was set to $40^{\circ} \mathrm{C}$ and the de-clustering potential in the ion 
funnel system was set to $0 \mathrm{~V}$. By these means ions are not relevantly de-clustered in the inlet part of the mass spectrometer. Helium is applied as gas in the collision cell and the ion collision energy in the collision cell is set to $2 \mathrm{~V}$, which establishes very soft collision conditions in the rear part of the spectrometer as well. It must be noted that the utilization of helium in the rear section of the mass spectrometer is crucial for detection of large protein clusters. The precise reason is currently not clear and could not be clarified with the manufacturer of the mass spectrometer who stated that the large protein species as detected e.g. in figure $\mathrm{s} 1$ should not be detectable by the mass spectrometer at all. Spectra rate was set to $0.3 \mathrm{~Hz}$ with a rolling average over two scans. Instrument setting of the mass spectrometer For reproduction of the experiments, figures $\mathrm{s} 8 \mathrm{a}-\mathrm{c}$ show screen shots of the instrument settings used. 


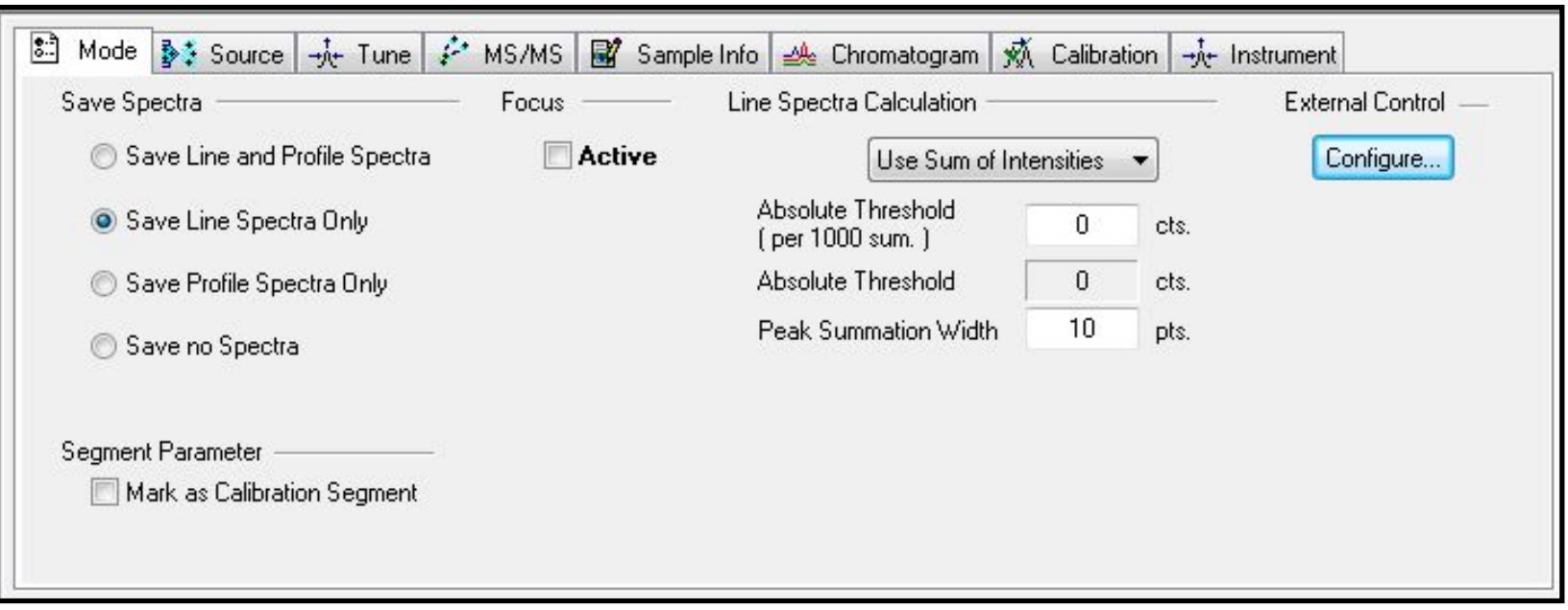

Figure s8a: Saving line spectra only limits the amount of data, even under this condition a mobility-classified mass spectrum has a size some hundred megabytes. Using the sum of intensities is favorable especially if isotopic patterns cannot be resolved for large species.

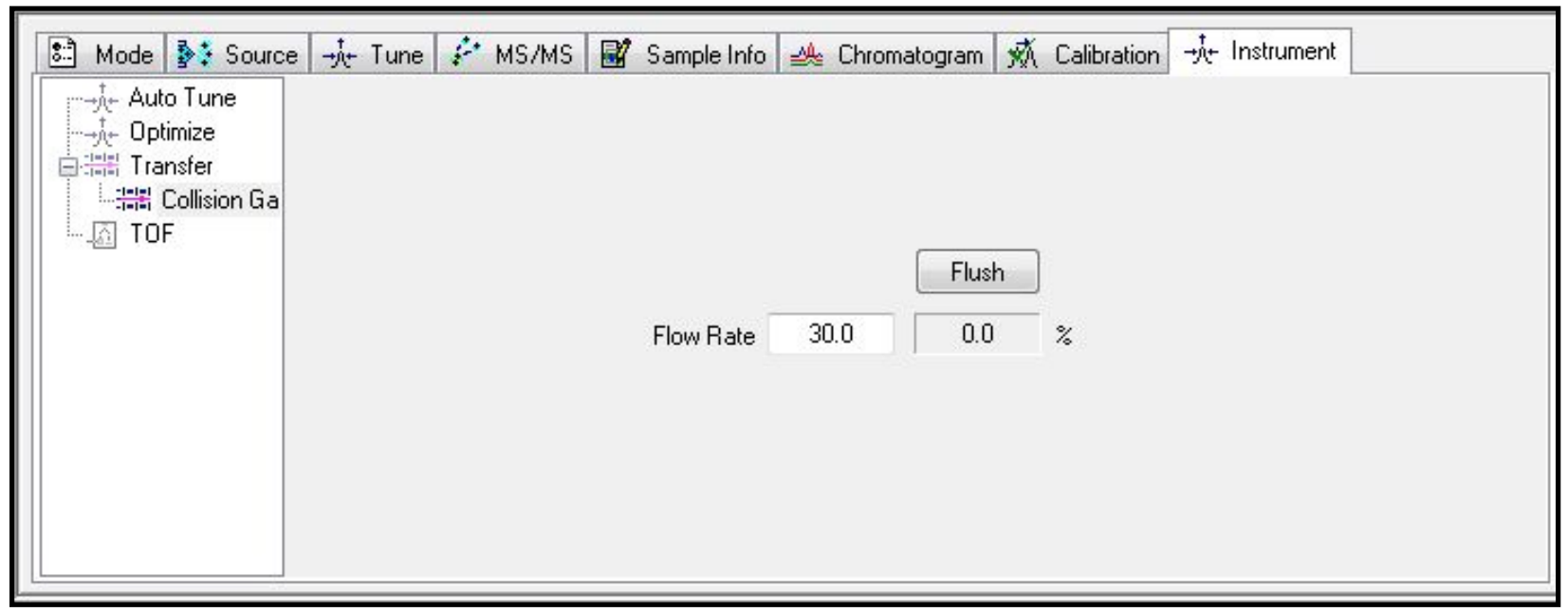

Figure s8b: Helium flow rate is set to $30 \%$ 


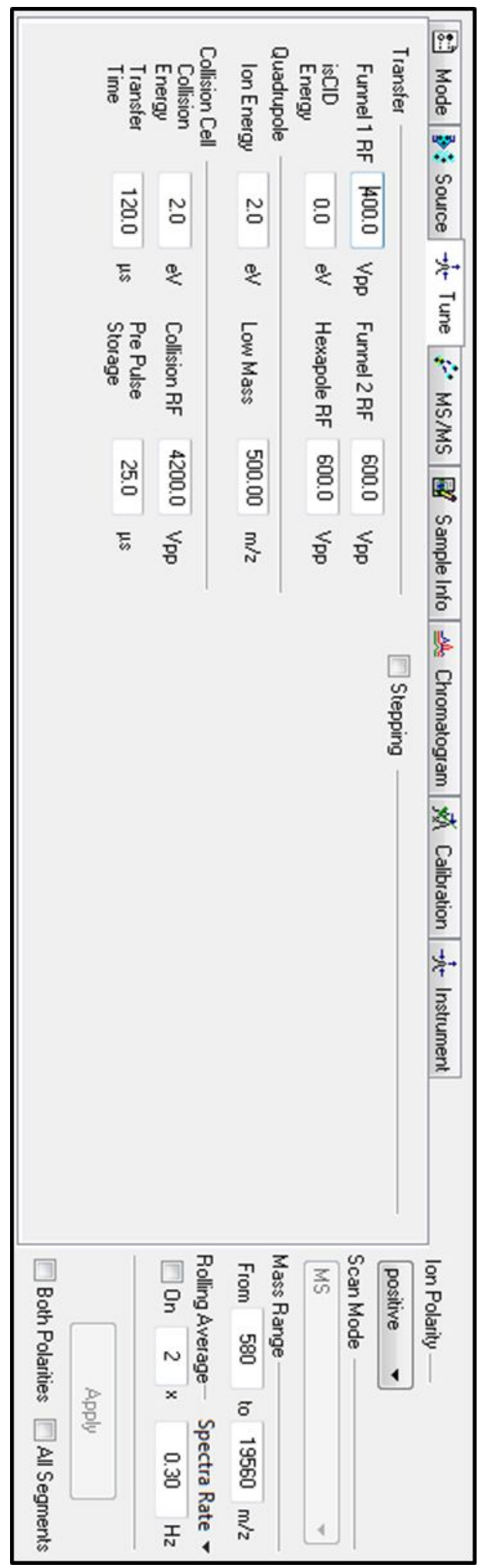

Figure s8c: Tuning parameters used. Same parameters are in the positive and negative ion mode. Polarity was only changed by the "Ion Polarity"-button. 


\section{S3: Electrospray setup and prediction of the cone-jet-mode scaling laws}

During review of the manuscript a question on the impact of proper cone-jet-mode operation on the outcome of an electrospray was asked. We think the answer to this question is a good starting point for this section.

Figure s9 shows a comparison of DMA-MS spectra recorded in the cone-jet-mode and in the intermittent cone-jet mode at a voltage just a little bit below of the cone-jet-mode voltage. While the residues of the parent droplets are visible in both spectra, the attribution of a certain size to this residue fraction is hardly possible for the intermittent cone-jet-mode. For narrowly distributed and thus best-defined boundary conditions of the ESI-process proper cone-jet mode operation is strongly recommended.
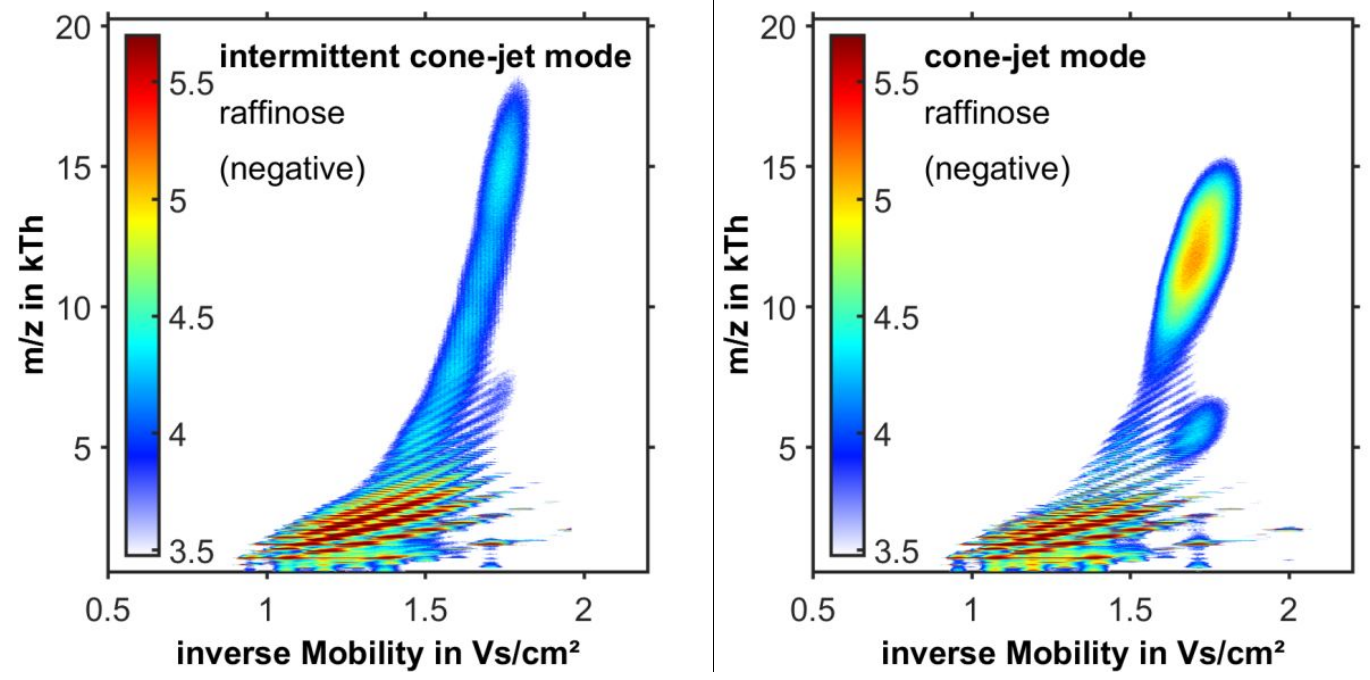

Figure s9: Comparison of DMA-MS spectra recorded in the intermittent cone jet mode and in the cone jet mode. 
The investment in a ESI-source that is reliably working in the cone-jet-mode (a mobility scan for the results in figure 1 or 9 takes $20 \mathrm{~min}$ and a single short failure of cone jet mode operation will more or less waste the scan) is thus clearly of great value.

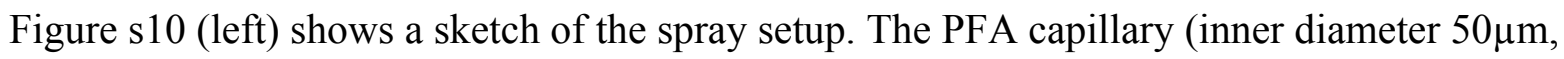
outer diameter $360 \mu \mathrm{m}$ ) is located on the axis of a sheath flow tube with outer diameter $2.1 \mathrm{~mm}$ and inner diameter $1.8 \mathrm{~mm}$. Solvent-saturated gas flows through the sheath flow tube surrounding the Taylor cone (s5(right)). Solvent evaporation from the Taylor cone is reduced and absorption of the organic vapor components by the Taylor cone liquid reduces the surface tension sufficiently to allow spraying in positive and negative polarity. For details on the approach see ${ }^{1}$.
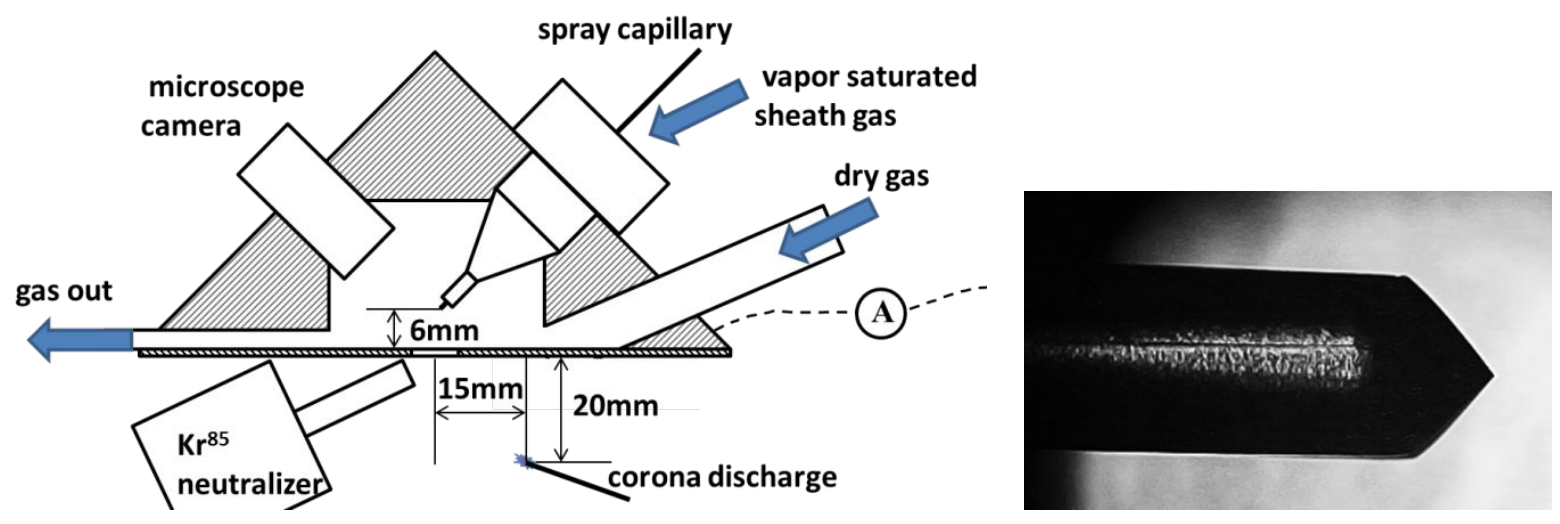

Figure s 10: Left: Spray configuration for the SMPS experiments, for DMA-MS a smaller version of the same source (without any charge neutralization) is used, right: Microscope image of the cone-jet mode during sampling.

With confirmed cone-jet mode operation, spray properties can be predicted by corresponding scaling laws. Based on the measurements of the dielectric constant effect by Chen and Pui the droplet size can be estimated as 


$$
d=3.7\left(\frac{\varepsilon_{0} \dot{V}}{K}\right)^{\frac{1}{3}}
$$

This result is obtained if the permittivity dependent droplet size pre-factor is approximated by 3.7 $\varepsilon_{r}^{-1 / 3}$, which matched the function given in ${ }^{2}$ with an error of less than $5 \%$ for relative permittivities above 35 .

The current uptake can be simplified in this permittivity range by

$$
I=2.35(\sigma K \dot{V})^{\frac{1}{2}}
$$

with an error of less than 5\% compared to the suggested correlation in the relative permittivity range above 35 as well. While equations S3-1 and S3-2 (as well the original functions given in ${ }^{2}$ ) describe the reality not perfectly precise, these scaling laws are a very sound basis for estimation of spray properties in the cone-jet mode. Validity of these equations is limited further to a certain range of the flow rate parameter

$$
\alpha=\frac{\rho_{L} K \dot{V}}{\varepsilon_{0} \varepsilon_{r} \sigma}
$$

Cone-jet mode operation with high permittivity liquids is (roughly) limited to a range of flow rate parameters $1<\alpha<10$. While the current uptake at a fixed flow rate parameter is independent of the liquid conductivity, many relevant factors do indeed depend on the liquid conductivity. 
For water as relevant solvent especially in biological applications we give properties for a flow rate parameter of 4 , for which cone jet mode operation is easily possible (if not prohibited by the occurrence gas discharge with large emitter dimensions as used here). Figure s11 shows all parameters (required liquid flowrate, droplet emission frequency, droplet diameter and the analyte concentration at which one expects by average a single molecule in each droplet) as function of the liquid conductivity.

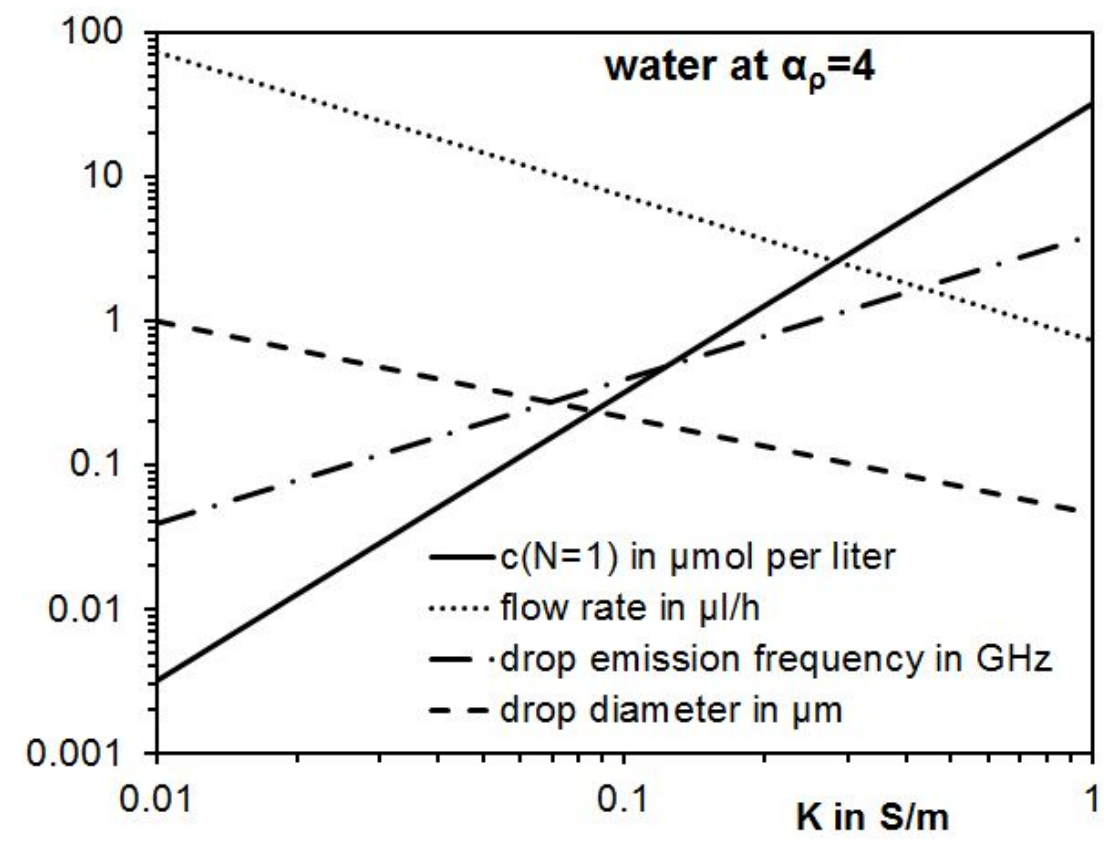

Figure s11: Required liquid flow rate, droplet emission frequency, droplet diameter and the analyte concentration at which one expects by average a single molecule in each droplet as function of liquid conductivity for water sprayed in the cone jet mode at a flow rate parameter of 4.

Using concentrations smaller than $\mathrm{c}(\mathrm{N}=1)$ will safely result in a very high singularization efficiency of the analyte. This is required for a quantitative analysis. As the number of analyte molecules transferred under such conditions (say a molecule in every $10^{\text {th }}$ droplet) is proportional to the emission frequency, good signal intensity requires high emission frequency and therefore 
high liquid conductivities. Additionally, the path a droplet travels before it is completely evaporated decreases with decreasing droplet size. Water droplets of $100 \mathrm{~nm}$ diameter have at ambient temperature and 50\% relative humidity a lifetime of only around 50 $\mu$ s. Such droplets are evaporated after a distance of a few $\mathrm{mm}$ from the Taylor cone tip. The spray can be placed very close to the MS inlet which guaranties high transfer efficiency.

\section{A comment on electrochemistry}

Employing electrochemistry-free conditions is comfortable (if one is not especially interested in the electro chemical reaction itself) as the electrochemical reaction bears in general the risk of sample altering or contamination. This is clear as the droplets generated in the cone-jet mode have typically a charge level of around $70 \% \ldots 90 \%$ of the Rayleigh-limit, which is given by:

$$
Q=2 \pi \sqrt{2 \varepsilon_{0} \sigma d_{d}^{3}}
$$

For instance, a droplet of $160 \mathrm{~nm}$ in diameter and a surface tension of $24 \mathrm{mN} / \mathrm{m}$ is charged to $70 \%$ of the Rayleigh-limit and carries around 1150 elementary charges or around $0.9 \mathrm{mmol}$ of charges per liter. These charges are generated in an electrochemical reaction at the location where the electric current enters the liquid. The fraction of the total electrolyte converted in the electrochemical reaction can be $>50 \%$ in extreme cases (water + acid). The electrochemical reaction may lead to sample altering either directly or indirectly by reactions of the analyte with the products of the electrochemical reaction or it might be a source of sample contamination due to generation of non-volatile reaction products as we have shown previously ${ }^{3}$. For this reason we 
employ an electrochemistry-free liquid feed technology as developed at our institute. A single protein, which has left the parent droplet neither in a Coulomb fission nor by ion evaporation, can easily be detected as evaporation residue of the parent droplet. This raises concerns about the electrochemical reaction as such a protein might react or cluster with reaction products of the electrochemical reaction if these were not all chemically inert and volatile.

\section{Electrospray operation in the lysozyme dilution series}

A stock solution of $1 \%$ (by volume) formic acid in water was prepared. The conductivity

of this solution was measured to be $0.308 \mathrm{~S} / \mathrm{m}$ by a conductometer (WTW Weilheim) and

a pH of 2.25 was measured by Mettler Toledo FE20 pH-meter. The solution was

sprayed at a current of $80+/-3 \mathrm{nA}$ (multi-meter in $\mathrm{DC}$ voltage mode, $1 \mathrm{M} \Omega$ inner resistivity)

under a vapor saturated sheath gas flow of $0.11 /$ min as adjusted by a rotameter. The

corresponding liquid flow rate is around $2.8 \mu \mathrm{l} / \mathrm{h}$ as derived from pressure-flow-rate

calibration of the spray capillary. The liquid composition in the saturators was water/2-

propanol 2:1 by mass. It has been shown ${ }^{1}$ that under such conditions the solvent

composition in the Taylor cone is very close to the liquid composition in the saturators

due to a very efficient mass transfer between the Taylor cone and the surrounding vapor

saturated gas phase. The absorption of 2-propanol from the gas phase leads to a 
significant change in conductivity. For that reason we have measured the conductivity of

$1 \%$ formic acid in water/2-propanol $2: 1$ by mass as well. The measured conductivity of $0.069 \mathrm{~S} / \mathrm{m}$ reflects the conductivity of the liquid in the Taylor cone much better than the conductivity of the feed liquid. The lowered surface tension $(0.024 \mathrm{~N} / \mathrm{m}$ for the water $/ 2$ propanol 2:1-mixture, measured by bubble pressure tensiometer, BP100, Krüss) allows spraying in the positive and negative cone jet mode.

From the liquid properties in the spray cone and the liquid flow rate, scaling laws (eq. S3-1,S3-2,S3-3) predict a droplet diameter of $172 \mathrm{~nm}$ and a current uptake of $84 \mathrm{nA}$. These values are in reasonable agreement with the measured current uptake of $80 \mathrm{nA}$ and a measured droplet size (see below) of 163nm.

\section{S4 Droplet size analysis by scanning mobility particle sizer (SMPS)}

For the analysis of droplet size spectra we follow the approach of Chen and Pui ${ }^{2,4}$.

Sucrose is added to the feed solution in a known volume concentration $(0.1 \ldots 1 \% \mathrm{~V})$.

From the evaporation residue size distribution of the remaining sugar particles one can estimate the primary droplet diameter 


$$
d_{d}=d_{r e s}\left[c_{v}\left(1-F_{o f f}\right)\right]^{-\frac{1}{3}}
$$

[S4-1]

As a certain volume fraction $F_{\text {off }}$ of the sucrose is found in the off-spring droplets the droplet size values as obtained by eq. S4-1 are corrected for this mass loss. The derived normalized number distribution of the parent droplets is shown in figure 3 in the manuscript for the conditions as employed in the dilution experiment (feed: water with $1 \% \mathrm{~V}$ formic acid, $\mathrm{pH} 2.25$, sprayed under vapor saturated nitrogen, liquid in saturators water/2-propanol 2:1 by mass, saturated nitrogen flow rate $1.5 \mathrm{~cm}^{3} / \mathrm{s}$, spray current $80 \pm 3 n A, 0.1 \%$ sucrose by volume). The calculated count-based modal diameter of the droplets is $163 \mathrm{~nm}$ and the excellent fit of the parent droplet mode by a normal distribution reveals a coefficient of variation of 0.10 .

The approach assumes sucrose evaporation residues that are dense, spherical and have the same density as the sucrose crystals. If one of these assumptions does not hold true, the calculated droplet size would be somewhat smaller compared to the calculated value. 


\section{S5: Optimization of particle transfer into the DMA}

To obtain a reasonable transmission for the large protein cluster ions with relatively low mobility, we have re-designed the DMA inlet situation. One basic problem with analyzing low mobility ions originates from the fact that the gas (nitrogen) that is sucked into the mass spectrometer is added to the DMA gas cycle. To prevent that solvent vapors and uncharged species entering the DMA gas cycle and a sucked into the mass spectrometer, the nitrogen flow rate into the DMA gas cycle has to be set somewhat higher than the flow rate sucked into the mass spectrometer such that a small gas flow is leaving the DMA though the ion entrance slit, as well. lons to be analyzed must therefore be transported into the DMA by drifting in the electric field faster than the counter flow gas velocity. The resulting mobility high pass problem is significantly reduced by the installation of a second slit orifice opposing the inlet orifice (figure s12, left). Insulated mounted this orifice can be put set on high voltage to establish an electric field that pushes low mobility ion effectively into the DMA. Sucking out a gas flow approximately twice as high as the outflow of the DMA creates additionally a stagnation 
point flow between the DMA inlet slit and the new additional orifice plate, which

transports the ions convectively very close to the DMA entrance. The newly created

situation is between the two slit orifices at the inlet is depicted in figure s8, right.
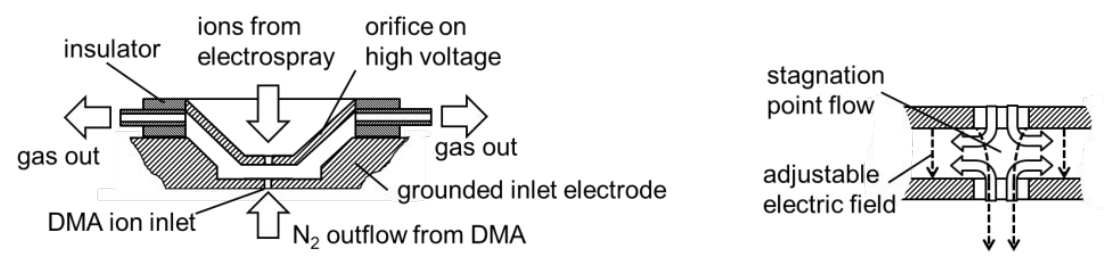

Figure s12: Left: Re-designed inlet situation of the DMA. Right: Electric field and gas flow situation at the DMA-inlet.

The higher transfer efficiency ${ }^{5}$ into the DMA results in a reduced resolving power of the DMA as the field strength inside the DMA is now much lower than the electric field generated between the orifices at the inlet. At the low gas velocity conditions used in the presented experiments resolving power is 24 for $\mathrm{THA}^{+}$-ion (tetraheptylammonium-ion) used for calibration. Gaussian-shaped transmission curves and increasing resolving power with increasing flow velocity indicate diffusion-limited resolution. As the residence time in the classifier is equal for all classified species, the theoretical diffusion-limited resolving power scales simply with the inverse square root of the diffusion coefficient of the classified species. The diffusion coefficient can be calculated directly from measured 
ion mobilities by the Einstein-Smoluchowski-correlation e.g. $D=Z k_{B} T /(z e)$ and therefore $R \propto \sqrt{z e /\left(Z k_{B} T\right)}$. The observed proteins and protein clusters (figure s14) carry at least 4 elementary charges and have at this low charge levels a mobility which is relevantly lower compared to that of the $\mathrm{THA}^{+}$-ion. If protein mobility resolution is diffusion limited, one would therefore expect a resolving power of at least 50 for the proteins observed. Practically, we find - in line with findings of $\operatorname{Hogan}^{6}$ - a resolving power in the order of 30 at best (31 for the lysozyme monomer in charge state -4$)$. As the DMA is capable of reaching resolving powers in excess of 60 the relatively low resolution of the protein peaks must be attributed to different protein conformations in the gas phase during classification.

\section{S6: DMA operation principle, operation parameters and calibration}

Figure s13 shows a scheme Seadm's planar DMA. Ions enter the DMA through a slit in the grounded front electrode voltage applied to the backside electrode creates an electric field between the inlet and the outlet side of the DMA in which the ions are drifting. Due to a gas flow between the inlet and the outlet electrode ions are convectively transported in the flow direction. With a fixed gas flow rate ion trajectories depend only on the applied voltage. The mobility of the ions reaching the exit slit from where they get into the mass spectrometer is inversely 
proportional to the applied voltage. As the flow velocity in the DMA is not known a one point calibration is required for the ion mobility.

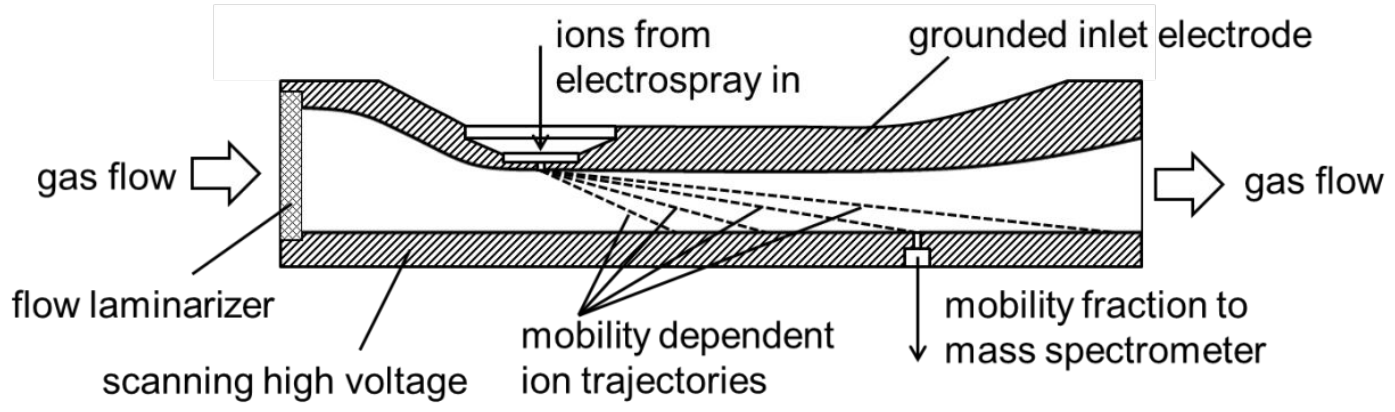

Figure s13: Scheme of Seadm's palar DMA as used in the DMA-MS experiments.

\section{Mobility analysis by DMA}

Voltage scans in the protein cluster measurements were performed starting at a voltage of $U\langle t=0\rangle=300 \mathrm{~V}$. For the raffinose and sodium chloride scans the staring voltage was stet o $0 \mathrm{~V}$. The voltage was steadily increased at a rate of $d U / d t=0.5 \mathrm{~V} / \mathrm{s}$.

As the flow rate in the DMA gas cycle is not directly measured but a constant gas flow is realized by control of turbo pump speed a one point calibration is required to be able to attribute mobilities to the detected ion peaks. Calibration of the system was performed using THA+ as mobility standard. Under the employed operation conditions (gas temperature in the DMA: $25^{\circ} \mathrm{C}$, pump speed: $\left.7000 \mathrm{rpm}\right)$ the $\mathrm{THB}+$ ion swept out at a 
voltage of $U_{T H A+}=413.6 \mathrm{~V} \pm 0.9$. From the known electrical mobility $Z_{T H A+}=1.01 \mathrm{~cm}^{2}$ $/ V s^{7}$ the mobility $Z$ of an ion passing the DMA at a voltage $U$ can be calculated as $Z=$ $Z_{T H A+} U_{T H A}+/ U$. From the time dependent voltage applied to the DMA $U\langle t\rangle=U\langle t=0\rangle+$ $d U / d t \cdot t$ one can attribute an ion mobility passing the DMA to every time in a mobility scan. As the voltage is scanned typically linearly and the electrical mobility of an ion species passing the DMA at a given voltage $U$ is inversely proportional to the value of the voltage $U \propto 1 / Z$ one finds

$$
\frac{1}{Z}\langle t\rangle=\frac{1}{Z_{T H A+} U_{T H A+}}\left[\frac{d U}{d t} t+U\langle t=0\rangle\right]
$$

[S6-1]

Mobility values given here are based on calibration values we have recorded. It must be noted that while the DMA gas temperature is kept controlled and constant, the DMA is a system open to ambient pressure. Common changes in ambient pressure $(980 \ldots 1020 \mathrm{kPa})$ have a direct and in the free molecular regime proportional impact on the ion mobility. Relaying on a single pressure mobility calibration may therefore on different measurement days result in deviations between measured electrical mobilities 
of possibly up to $4 \%$. As the very exact determination of mobility values is, however, not the scope of this work this error is judged to be acceptable.

\section{S7: Monomer spectra}

Figure s14 shows mobility-averaged mass spectra using the MS-software included averaging function. Only the monomers are visible in these averaged mass spectra. For the monomer signal intensity in figure 8 in the main manuscript we have summed up the intensities of charge states 79 in positive ion mode and 4-6 in the negative ion mode. Intensities were considered for the whole isotopic patterns.

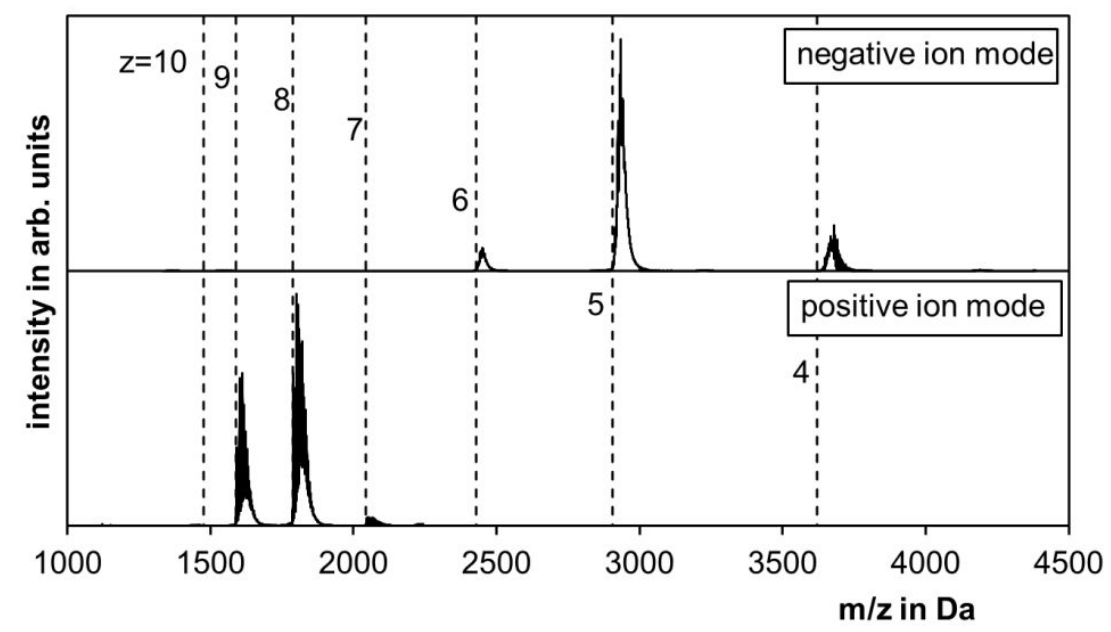

Figure s14: Mobility-averaged mass spectra show monomer peaks only. Relevant abundances are observed for only two charge stages in both polarities. The charge in the positive ion mode is twice as high (+9 and +8$)$ as the charge in the negative ion mode (-5 and -4$)$. 


\section{References}

(1) Lübbert, C.; Peukert, W. The mass transfer at Taylor cones. Journal of Aerosol Science 2018, 123, $39-51$.

(2) Chen, D.-R.; Pui, D. Y. H. Experimental Investigation of Scaling Laws for Electrospraying: Dielectric Constant Effect. Aerosol Science and Technology 1997, 27, 367-380.

(3) Lübbert, C.; Peukert, W. How to avoid interfering electrochemical reactions in ESI-MS analysis. Journal of mass spectrometry: JMS 2019, 54, 301-310.

(4) Chen, D.-R.; Pui, D. Y. H.; Kaufman, S. L. Electrospraying of conducting liquids for monodisperse aerosol generation in the $4 \mathrm{~nm}$ to $1.8 \mu \mathrm{m}$ diameter range. Journal of Aerosol Science 1995, 26, 963-977.

(5) Domaschke, M.; Lübbert, C.; Peukert, W. Analysis of ultrafine metal oxide particles in aerosols using mobility-resolved time-of-flight mass spectrometry. Journal of Aerosol Science 2019, 137, 105438.

(6) Hogan, C. J.; Fernández de la Mora, J. Ion mobility measurements of nondenatured 12-150 kDa proteins and protein multimers by tandem differential mobility analysis-mass spectrometry (DMA-MS). Journal of the American Society for Mass Spectrometry 2011, 22, 158-172.

(7) Fernández de la Mora, J.; Thomson, B. A.; Gamero-Castaño, M. Tandem mobility mass spectrometry study of electrosprayed tetraheptyl ammonium bromide clusters. Journal of the American Society for Mass Spectrometry 2005, 16, 717-732. 